\title{
Conservation Laws and Stress-energy-momentum Tensors for Systems with Background Fields
}

\author{
Jonathan Gratus*, \\ Lancaster University, Lancaster LA1 4YB, UK and \\ The Cockcroft Inistitute, Daresbury Laboratory, Warrington WA4 4AD, UK \\ Yuri N Obukhov ${ }^{\dagger}$ \\ Institute for Theoretical Physics, University of Cologne, 50923 Köln, Germany \\ Robin W Tucker ${ }^{\ddagger}$ \\ Lancaster University, Lancaster LA1 4YB, UK and \\ The Cockcroft Inistitute, Daresbury Laboratory, Warrington WA4 4AD, UK
}

October 15, 2018

\begin{abstract}
This article attempts to delineate the roles played by non-dynamical background structures and Killing symmetries in the construction of stress-energy-momentum tensors generated from a diffeomorphism invariant action density. An intrinsic coordinate independent approach puts into perspective a number of spurious arguments that have historically lead to the main contenders, viz the Belinfante-Rosenfeld stress-energy-momentum tensor derived from a Noether current and the EinsteinHilbert stress-energy-momentum tensor derived in the context of Einstein's theory of general relativity. Emphasis is placed on the role played by non-dynamical background (phenomenological) structures that discriminate between properties of these tensors particularly in the context of electrodynamics in media. These tensors are used to construct conservation laws in the presence of Killing Lie-symmetric background fields.
\end{abstract}

keywords Noether currents, Abraham stress-energy-momentum tensor, Minkowski stress-energy-momentum tensor,

Canonical stress-energy-momentum tensor,

Einstein-Hilbert stress-energy-momentum tensor,

Diffeomorphism invariance

MSC 70S10, 83C50, 70S05, 78A25, 83C05

*j.gratus@lancaster.ac.uk, Corresponding Author

†yo@thp.uni-koeln.de

${ }_{\ddagger}^{\ddagger}$ r.tucker@lancaster.ac.uk 


\section{Introduction}

The venerable aim of the variational calculus as a tool for deriving from a single principle the observed laws of physics remains an attractive one. It synthesises conservation laws from local symmetries and offers a route for finding unified schemes that underpin the experimental sciences. However the epistemology that arises in this approach has, on occasion, lead to unnecessary confusion when employed in the discussion of systems that require phenomenological input. In particular this remains true in the context of electrodynamics in macroscopic material where the indiscriminate use of the stressenergy-momentum concept in classical physics has lead to different notions of quantum electrodynamics in such media. The calculus often enables observed local and global symmetries of nature to be accommodated in a natural manner and can be used to generate conserved currents associated with local symmetries. Furthermore the local dynamics of coupled systems follows from local extrema of a functional constructed from a single diffeomorphism invariant action integral $S=\int_{\mathcal{M}} \Lambda^{\mathbf{T}}$, for some diffeomorphism invariant differential 4-form $\Lambda^{\mathbf{T}}$ on spacetime $\mathcal{M}$. If one chooses a nowhere vanishing 4-form $\Omega$ on spacetime and writes $\Lambda^{\mathbf{T}}=\mathcal{S} \Omega$ then $\mathcal{S}$ is called a scalar action density (relative to $\Omega$ ). For any covariant metric $g$ on $\mathcal{M}$ a natural choice is $\Omega=\sqrt{|\operatorname{det} g|} d^{4} x=\star 1$ where $\star$ is the Hodge map associated with $g$. An alternative choice, given a coordinate system $\left(x^{1}, \ldots, x^{4}\right)$ is the tensor density $\Omega=d x^{1} \wedge \cdots \wedge d x^{4}$. Such a choice implies that $\mathcal{S}$ then transforms like $|\operatorname{det} g|^{-1 / 2}$ under a local change of coordinates 1 .

Traditional symmetry analysis based on the pioneering work of Noether exploits such densities often in a manifestly coordinate dependent manner. Modern approaches exploit an intrinsic jet-bundle formulation. Between these extremes are ad-hoc formulations that have led to intense debates about the role of alternative stress-energy-momentum tensors used to describe the interchange of energy and momentum between interacting systems in continuous macroscopic media. In particular, conserved Noether currents have historically been constructed from 3-forms on space and their associated Lagrangian scalar densities. Notions of stress and power in continuum mechanics became incorporated into the Lorentz covariant formulation of special relativity. With the advent of Einstein's general theory of relativity the spacetime symmetric metric tensor field becomes a dynamical variable and an alternative stress-energy-momentum tensor can be defined through the variational derivative of the action 4-form on spacetime (for gravity and matter) with respect to this metric tensor 2 The issue that then arises is how best to identify conserved quantities constructed from different models that can be put into a variational formulation and relate them to different choices of stress-energy-momentum tensor.

Conserved quantities can be generated from closed 3-forms in spacetime. These often arise as a consequence of some symmetry of an element or elements contributing to the structure of the action for the model. However even in the absence of such symmetries there are powerful relations that arise from applying the variational approach to action functionals that are (locally) invariant under transformations of the fields induced by arbitrary (local) spacetime diffeomorphisms. Such functionals are readily constructed in

\footnotetext{
${ }^{1}$ If $U=\partial / \partial x^{1}$ generates time translations then $i_{U} \Lambda^{\mathbf{T}}$ may be called a Lagrangian 3-form 4 relative to $U$.

${ }^{2}$ Matter in this sense includes electromagnetism as well as other matter fields. Modified theories of gravity, e.g. with scalar fields coupled directly to spacetime curvature, offer no unique identification of a "matter" action.
} 
terms of coordinate invariants made by contracting tensor fields of various degrees or tensor "densities" of different weights. It is not necessary that all such quantities in this construction be dynamical, i.e. subject to variational field equations. However it is in these circumstances that there arise differences when one compares the consequences deduced from different choices for the description of stress, energy, and momentum in the presence of such quantities.

The (covariant) variational approach is often restricted to closed (non-dissipative) systems where dynamical equations for the dynamical field variables arise by finding local extrema of a total action $S$ under their variations, where $\Lambda^{\mathbf{T}}$ is a 4 -form on a spacetime manifold. Such forms belong to a class, members of which describe the same classical physics. For example members that differ by an exact 4-form with compact support yield the same variational equations.

Since the description of gravitation is given in terms of a geometry of spacetime, any collection of dynamical field variables $\{\boldsymbol{\zeta}\}=\left\{\zeta_{1}, \ldots, \zeta_{Q}\right\}$ that are not part of the geometry may be assigned the status of "matter" 3 In Einstein's theory, gravitation arises from a pseudo-Riemannian spacetime geometry based entirely on a dynamical spacetime metric tensor field $g$. In the absence of matter, the dynamics of $g$ is given by the Einstein-Hilbert action $\int_{\mathcal{M}} \Lambda^{\text {Ein }}$. The dynamics of (non-spinorial) matter minimally coupled to gravitation is described by a matter action 4 -form $\Lambda^{\mathrm{m}}$ that is independent of derivatives of $g$.

In order to eliminate use of the jet-bundle language and simplify the analysis, in the following the matter action 4 -form $\Lambda^{\mathrm{m}}$ will be restricted to depend on $g,\{\boldsymbol{Z}\},\{\boldsymbol{\zeta}\}$ and $\{d \boldsymbol{\zeta}\}$, where a collection of prescribed non-dynamical background tensor fields $\{\boldsymbol{Z}\}=$ $\left\{Z_{1}, \ldots, Z_{N}\right\}$ has been included. Such fields play no variational role in fixing the local extrema of $S$ but (if present) play a crucial role in determining the consequences that follow from diffeomorphism invariance. Then the total action 4-form is $\Lambda^{\mathbf{T}}=\Lambda^{\text {Ein }}+\Lambda^{\mathbf{m}}$.

It is sometimes useful to define matter subsystems that are described by sub-actions $\int_{\mathcal{M}} \Lambda^{s}$ where $\Lambda^{\mathrm{m}}=\sum_{s} \Lambda^{s}$. Clearly this notion of a subsystem is defined relative to a particular decomposition of $\Lambda^{\mathrm{m}}$ and extrema of $S$ will not in general coincide with extrema of $\int_{\mathcal{M}}\left(\Lambda^{\text {Ein }}+\Lambda^{s}\right)$. When such extrema do coincide one may argue that the subsystem described by $\Lambda^{s}$ decouples from the system described by $\Lambda^{\mathbf{T}}$, i.e. it becomes a closed subsystem. This is rarely the case in systems interacting with dynamical gravitation. Subsystems that are not closed are termed open.

Einstein's gravitational field equations in the presence of matter lead one to identify the variational derivative of $\Lambda^{\mathrm{m}}$ with respect to $g$ (see below) with the symmetric "stress-energy-momentum" tensor $T^{\mathcal{H}}(g,\{\boldsymbol{Z}\},\{\boldsymbol{\zeta}\},\{d \boldsymbol{\zeta}\})$ associated with matter. This terminology is natural, given its historic connection with Newtonian concepts but arguably misleading in a broader context where symmetries associated with space and time translation are absent. The tensor $T^{\mathcal{H}}$ relies for its definition on $\Lambda^{\mathrm{m}}$ depending on the spacetime metric. On manifolds where gravitation is irrelevant (where $g$ is regarded as a non-dynamical prescribed background) or no preferred metric is available one may find alternative approaches leading to conserved quantities. In such circumstances there exist 3-forms derived from a diffeomorphism invariant actions $\int_{\mathcal{M}} \Lambda^{\mathrm{m}}$ that give rise to certain vector valued maps on vector fields. These give rise to two other maps $T^{\mathcal{N}}$ and $T^{\mathcal{B}}$ which have traditionally been associated with "canonical stress-energy-momentum tensors" and are related by the so called Belinfante-Rosenfeld procedure. It is shown below that these

\footnotetext{
${ }^{3}$ In view of the ambitions of M-theory this interpretation may be an effective one imposed by dimensional reduction.
} 
give rise to conserved currents in the presence of background fields which possess appropriate Lie-symmetries and that $T^{\mathcal{H}}$ gives rise to conserved currents, in general, only if $g$ is dynamical and satisfies Einstein's theory of general relativity. Furthermore it is demonstrated that any difference between $T^{\mathcal{B}}$ and $T^{\mathcal{H}}$ arises from the dependence of $\Lambda^{\mathbf{m}}$ on the background fields $\{\boldsymbol{Z}\}$. In this article the consequences of diffeomorphism invariance of matter actions on all these quantities will be explored.

The formalism below first establishes an intrinsic variational calculus for actions involving the metric tensor field $g$, arbitrary tensor fields $\{\boldsymbol{Z}\}$ and differential forms $\{\boldsymbol{\zeta}\},\{d \boldsymbol{\zeta}\}$ on an $n$-dimensional manifold $M$. Any differential form of degree $n$ on $M$ will be called a top-form. The set of all $p$-form fields is written $\Gamma \Lambda^{p} M$. Thus the matter action is some top-form:

$$
\Lambda^{\mathbf{m}}\left(g, Z_{1}, \ldots, Z_{N}, \zeta_{1}, d \zeta_{1}, \ldots, \zeta_{Q}, d \zeta_{Q}\right) \in \Gamma \Lambda^{n} M
$$

and depends in general on

- a metric tensor field $g$,

- a collection of mixed degree tensor fields (including scalar fields) of no particular symmetry $Z_{A}$ for $A=1, \ldots, N$

- a collection of differential forms $\zeta_{B} \in \Gamma \Lambda^{p_{B}} M$ for $B=1, \ldots, Q$ and their exterior derivatives $d \zeta_{B}$. These fields will be taken to satisfy variational field equations following from some action top-form $\Lambda^{\mathrm{m}}$. Since $\Lambda^{\text {Ein }}$ is independent of $Z_{A}$ and $\zeta_{B}$ these are the same as the variational equations that follow from the action top-form $\Lambda^{\mathbf{T}}$.

The notation used in the paper is given in section 1.1. In section 2 partial Gateaux derivatives of $\Lambda^{\mathrm{m}}$ with respect to tensors and differential forms are related and compared with standard partial variational derivatives using an intrinsic formulation. Diffeomorphism invariance of $\Lambda^{\mathrm{m}}$ is defined and its consequences expressed in terms of certain maps $\mathcal{A}$ and $\mathcal{B}$ on these derivatives. In section 3 the Einstein-Hilbert and other stressenergy-momentum tensors are defined and expressed in terms of these maps. Relations are derived between these tensors and tensor densities when all matter fields satisfy the variational field equations derived from $\Lambda^{\mathrm{m}}$. It is then shown how conserved quantities can arise in the presence of material and Killing Lie-symmetric background fields. In the concluding section the physical implications of these relations are emphasized. The Appendix gives some mathematical details and proofs of results used in the main text.

\section{$1.1 \quad$ Notation}

Local coordinates on $M$ are denoted $\left(x^{1}, \ldots, x^{n}\right)$. These define a local coordinate frame $\left\{\partial_{1}, \ldots, \partial_{n}\right\}$ where $\partial_{a}=\frac{\partial}{\partial x^{a}}$ and coordinate co-frame $\left\{d x^{1}, \ldots, d x^{n}\right\}$. In these frames a metric $g=g_{a b} d x^{a} \otimes d x^{b}$ and the inverse metric $\widetilde{g}=g^{a b} \partial_{a} \otimes \partial_{b}$ where $g^{a b} g_{b c}=\delta_{c}^{a}$. Here implicit summation is over $a, b=1, \ldots, n$. The metric dual of any vector field $v \in \Gamma T M$ is the 1 -form $\widetilde{v}=g(v,-) \in \Gamma \Lambda^{1} M$. In spacetime $n=4$ and $g$ is Lorentzian with signature $(-1,+1,+1,+1)$ here. A map $T: X \mapsto T(X)$ is $f$-linear if $T(f X)=f T(X)$ for all scalar fields $f \in \Gamma \Lambda^{0} M$. The map $T$ is $\mathbb{R}$-linear if $T(\lambda X)=\lambda T(X)$ for all constants $\lambda \in \mathbb{R}$. In the following the word "tensor" refers to an $f$-multilinear map on vectors and 
their duals (co-vectors). Given a non-vanishing top-form $\Omega$, the phrase " $\mathcal{T}$ is a tensor density with respect to $\Omega$ of weight $W$ " implies that when $\Omega$ is replaced by $\hat{\Omega}=J \Omega$ for $J \in \Gamma \Lambda^{0} M$ nowhere vanishing then $\mathcal{T}$ transforms to $\hat{\mathcal{T}}=J^{W} \mathcal{T}$. If $\Omega=d x^{1} \wedge \cdots \wedge d x^{n}$ and $\hat{\Omega}=d y^{1} \wedge \cdots \wedge d y^{n}$ are related by coordinate transformation then $J$ is the Jacobian of the transformation. Choosing $\Omega=\star 1$ one can convert $\mathcal{T}$ into a bona-fide tensor field $(\hat{\mathcal{T}}=\mathcal{T})$. The Lie derivative of any tensor $T$ with respect to any vector field $v$ on $M$ is denoted $\mathcal{L}_{v} T$ and the exterior derivative $d$ on differential forms is defined so that $d^{2}=0$. A form $\beta$ is said to be closed if $d \beta=0$ and exact if $\beta=d \alpha$ for some $\alpha$. The interior contraction operator with respect to $v$ on forms, denoted $i_{v}$, is a graded derivative and $\left(i_{v}\right)^{2}=0$.

\section{Intrinsic Variational Calculus}

\subsection{Algebraic Preliminaries}

The degree of an arbitrary tensor will be represented as an ordered list $\boldsymbol{s}$ of 0 or more entries. Each entry is either the symbol $\mathbb{F}$ (for 1 -form) or $\mathbb{V}$ (for vector) e.g. $s=[\mathbb{F}, \mathbb{V}, \mathbb{V}, \mathbb{F}]$. The bundle of tensors of degree $s$ over $M$ is denoted $\otimes^{s} M$, with sections in $\Gamma \otimes^{s} M$ and the bundles of 0 -forms, 1 -forms and vector fields are written

$$
\Lambda^{0} M=\otimes^{[]} M, \quad \Lambda^{1} M=\otimes^{[\mathbb{F}]} M \quad \text { and } \quad T M=\otimes^{[\mathbb{V}]} M
$$

respectively. Furthermore

$$
\left(\otimes^{s} M\right) \otimes\left(\otimes^{t} M\right)=\otimes^{[s, t]} M
$$

where $[\boldsymbol{s}, \boldsymbol{t}]$ is simply the concatenation of the two lists. Thus

$$
v \otimes \zeta \otimes u \in \Gamma \otimes \otimes^{[\mathbb{V}, \mathbb{F}, \mathbb{V}]} M
$$

where $v, u \in \Gamma T M$ and $\zeta \in \Gamma \Lambda^{1} M$. The metric $g$ lies in the symmetric sub-bundle of $\otimes^{[\mathbb{F}, \mathbb{F}]} M$ and the inverse metric $\tilde{g}$ lies in the symmetric sub-bundle of $\otimes^{[\mathbb{V}, \mathbb{V}]} M$. Similarly, since

$$
\sum_{I_{1}<\ldots<I_{p}} \alpha_{I_{1} \cdots I_{p}} e^{I_{1}} \wedge \cdots \wedge e^{I_{p}}=\frac{1}{p !} \sum_{I_{1}<\ldots<I_{p}} \sum_{\sigma \in S_{p}} \alpha_{I_{1} \cdots I_{p}} \epsilon(\sigma) e^{\sigma\left(I_{1}\right)} \otimes \cdots \otimes e^{\sigma\left(I_{p}\right)}
$$

where $S_{p}$ is the set of permutations of $\left\{I_{1}, \ldots, I_{p}\right\}$ and $\epsilon(\sigma)$ is the signature of the permutation, the $p$-form bundle $\Lambda^{p} M$ is the antisymmetric sub-bundle of $\otimes^{[\mathbb{F}, \ldots, \mathbb{F}]} M$ where $[\mathbb{F}, \ldots, \mathbb{F}]$ has length $p$.

The dual space of $\otimes^{s} M$ is $\otimes^{\bar{s}} M$ where $\bar{s}$ is obtained by interchanging the symbols $\mathbb{F}$ and $\mathbb{V}$ in $\boldsymbol{s}$. The total contraction of elements in $\Gamma \otimes^{\bar{s}} M$ with elements in $\Gamma \otimes^{s} M$ is written

$$
\Gamma \otimes^{\bar{s}} M \times \Gamma \otimes^{s} M \rightarrow \Gamma \Lambda^{0} M, \quad(\Phi, Z) \mapsto \Phi: Z
$$

where $\Phi \in \Gamma \otimes^{\bar{s}} M$ and $Z \in \Gamma \otimes^{s} M$. It is defined inductively via

$$
\zeta: v=v: \zeta=\zeta(v) \quad \text { where } \quad \zeta \in \Gamma \otimes^{[\mathbb{F}]} M \quad \text { and } \quad v \in \Gamma \otimes^{[\mathbb{V}]} M
$$


and extended by $f$-linearity to arbitrary tensors via

$$
\left(\Phi_{1} \otimes \Phi_{2}\right):\left(Z_{1} \otimes Z_{2}\right)=\left(\Phi_{1}: Z_{1}\right)\left(\Phi_{2}: Z_{2}\right)
$$

Thus for example

$$
\left(\zeta_{1} \otimes u \otimes \zeta_{2}\right):\left(v_{1} \otimes \beta \otimes v_{2}\right)=\zeta_{1}\left(v_{1}\right) \beta(u) \zeta_{2}\left(v_{2}\right)
$$

where $\left(\zeta_{1} \otimes u \otimes \zeta_{2}\right) \in \Gamma \otimes^{[\mathbb{F}, \mathbb{V}, \mathbb{F}]} M$ and $\left(v_{1} \otimes \beta \otimes v_{2}\right) \in \Gamma \otimes^{[\mathbb{V}, \mathbb{F}, \mathbb{V}]} M$.

Using this notation $\Lambda^{\mathbf{m}}$ may be regarded as a fibre bundle morphism

$$
\Lambda^{\mathbf{m}}: \mathcal{E}^{(g)} \oplus \mathcal{E}^{(Z)} \oplus \mathcal{E}^{(\zeta)} \rightarrow \Lambda^{n} M
$$

which we write $\Lambda^{\mathbf{m}}\left(g, Z_{1}, \ldots, Z_{N}, \zeta_{1}, d \zeta_{1}, \ldots, \zeta_{Q}, d \zeta_{Q}\right)$. Here

- $\mathcal{E}^{(g)}=\bigotimes^{[\mathbb{F}, \mathbb{F}]} M$ is the bundle of metrics, i.e. $g \in \Gamma \mathcal{E}^{(g)}$.

- $\mathcal{E}^{(Z)}=\otimes^{s_{1}} M \oplus \cdots \oplus \otimes^{s_{N}} M$ is the bundle of $N$ tensors of the appropriate degrees, i.e $\left(Z_{1}, \ldots, Z_{N}\right) \in \Gamma \mathcal{E}^{(Z)}$ where $Z_{A} \in \Gamma \otimes^{s_{A}} M$.

- $\mathcal{E}^{(\zeta)}=\Lambda^{p_{1}} M \oplus \Lambda^{p_{1}+1} M \oplus \cdots \oplus \Lambda^{p_{Q}} M \oplus \Lambda^{p_{Q}+1} M$ is the bundle of $Q$ pairs of forms of the appropriate degrees, i.e $\left(\zeta_{1}, d \zeta_{1}, \ldots, \zeta_{Q}, d \zeta_{Q}\right) \in \Gamma \mathcal{E}^{(\zeta)}$ where $\zeta_{B} \in \Gamma \Lambda^{p_{B}} M$.

Since $\Lambda^{\mathbf{m}}$ is a fibre bundle morphism, the value of $\Lambda^{\mathbf{m}}\left(g, Z_{1}, \ldots, Z_{N}\right.$,

$\left.\zeta_{1}, d \zeta_{1}, \ldots, \zeta_{Q}, d \zeta_{Q}\right)\left.\right|_{x}$ for some point $x \in M$ depends only on the values of its arguments at that point, i.e.

$$
\begin{aligned}
& \left.\Lambda^{\mathbf{m}}\left(g, Z_{1}, \ldots, Z_{N}, \zeta_{1}, d \zeta_{1}, \ldots, \zeta_{Q}, d \zeta_{Q}\right)\right|_{x}= \\
& \quad \Lambda^{\mathbf{m}}\left(\left.g\right|_{x},\left.Z_{1}\right|_{x}, \ldots,\left.Z_{N}\right|_{x},\left.\zeta_{1}\right|_{x},\left.d \zeta_{1}\right|_{x}, \ldots,\left.\zeta_{Q}\right|_{x},\left.d \zeta_{Q}\right|_{x}\right)
\end{aligned}
$$

In this language a model containing matter in a linear (temporally and spatially) nondispersive medium interacting with the electromagnetic field $F=d A$ is characterised by a $U(1)$ gauge invariant excitation tensor $G=\chi\left(g, Z_{1}, \ldots, Z_{N}, d A, \zeta_{2}, d \zeta_{2}, \ldots, \zeta_{Q}, d \zeta_{Q}\right)$ for some constitutive tensor $\chi$ and is described by an action 4 -form on spacetime $\mathcal{M}$

$$
\begin{aligned}
\Lambda^{\mathbf{m}}\left(g, Z_{1}, \ldots,\right. & \left.Z_{N}, A, d A, \zeta_{2}, d \zeta_{2}, \ldots, \zeta_{Q}, d \zeta_{Q}\right)= \\
& \frac{1}{2} F \wedge \star G+\Lambda^{\mathbf{Q}}\left(g, \zeta_{2}, D \zeta_{2}, \ldots, \zeta_{Q}, D \zeta_{Q}\right)
\end{aligned}
$$

where $D \xi=d \xi$ for electrically neutral real fields $\xi$ and is the $U(1)$ exterior covariant derivative for complex charged fields.

A particular model [2, 3] involving only a single non-dynamic tensor $Z$ together with $F$ and $g$ is described by the action 4-form $\Lambda^{\mathbf{m}}: \mathcal{E}^{(g)} \oplus \mathcal{E}^{(Z)} \oplus \mathcal{E}^{(\zeta)} \rightarrow \Lambda^{4} \mathcal{M}$ (with the bundles $\mathcal{E}^{(Z)}=\otimes^{[\mathbb{F}, \mathbb{F}, \mathbb{V}, \mathbb{V}]} \mathcal{M}, \mathcal{E}^{(\zeta)}=\Lambda^{2} \mathcal{M}$ over spacetime $\left.\mathcal{M}\right)$ :

$$
\Lambda^{\mathbf{m}}(g, Z, d A)=\frac{1}{4} F \wedge \star\left(Z(F)+Z^{\dagger}(F)\right)
$$

with

$$
F=d A
$$


and where $Z^{\dagger}$ is the adjoint of $Z$ defined by

$$
\alpha \wedge \star Z^{\dagger}(\beta)=\beta \wedge \star Z(\alpha)
$$

for all $\alpha, \beta \in \Gamma \Lambda^{2} \mathcal{M}$. Unlike $Z$, the tensor $Z^{\dagger}$ depends on $g$. Varying (5) with respect to $A$ then yields, in terms of the notation defined in (22) below

$$
\frac{\delta \Lambda^{\mathbf{m}}}{\delta A}(g, Z, d A)=0
$$

i.e.

$$
d \star G=0
$$

where in terms of (20) below, the excitation tensor $G \in \Gamma \Lambda^{2} \mathcal{M}$ is given by

$$
G=\star^{-1}\left(\frac{\partial \Lambda^{\mathbf{m}}}{\partial(d A)}\right)
$$

i.e

$$
G=\chi(F)=\frac{1}{2}\left(Z(F)+Z^{\dagger}(F)\right)
$$

Equations (6) and (86) constitute the "on-shell" Maxwell system for model (50) in any background $g, Z$. In this model the constitutive tensor $\chi$ is independent of the motion of the medium. Although from (7) $Z^{\dagger}$ depends on the metric it follows from (10) that (5) can be written

$$
\Lambda^{\mathbf{m}}(g, Z, d A)=\frac{1}{2} F \wedge \star Z(F)
$$

and thus the only metric dependence of $\Lambda^{\mathbf{m}}$ is through the Hodge map.

A more complex model [3] in which the constitutive tensor $\chi$ depends explicitly on the motion of the medium and exhibits intrinsic magneto-electric constitutive properties involves a timelike ${ }^{4}$ vector field $V$ and four background degree 2 tensors $Z^{\mathrm{de}}, Z^{\mathrm{db}}, Z^{\mathrm{he}}, Z^{\mathrm{hb}}$. It is described by the action 4-form $\Lambda^{\mathrm{m}}: \mathcal{E}^{(g)} \oplus \mathcal{E}^{(Z)} \oplus \mathcal{E}^{(\zeta)} \rightarrow \Lambda^{4} \mathcal{M}$ where $\mathcal{E}^{(Z)}=$ $\otimes^{[\mathbb{F}, \mathbb{V}]} \mathcal{M} \oplus \otimes^{[\mathbb{F}, \mathbb{V}]} \mathcal{M} \oplus \otimes^{[\mathbb{F}, \mathbb{V}]} \mathcal{M} \oplus \otimes^{[\mathbb{F}, \mathbb{V}]} \mathcal{M} \oplus \otimes^{[\mathbb{V}]} \mathcal{M}, \mathcal{E}^{(\zeta)}=\Lambda^{2} \mathcal{M}$

$$
\begin{aligned}
\Lambda^{\mathrm{m}}\left(g, Z^{\mathrm{de}}, Z^{\mathrm{db}}, Z^{\mathrm{he}}, Z^{\mathrm{hb}}, V, d A\right) & \\
=\frac{1}{2} F \wedge & \star\left(Z^{\mathrm{de}}\left(i_{V_{g}} F\right) \wedge \widetilde{V}_{g}+Z^{\mathrm{db}}\left(i_{V_{g}} \star F\right) \wedge \widetilde{V}_{g}\right. \\
& \left.-\star\left(Z^{\mathrm{he}}\left(i_{V_{g}} F\right) \wedge \widetilde{V}_{g}\right)-\star\left(Z^{\mathrm{hb}}\left(i_{V_{g}} \star F\right) \wedge \widetilde{V}_{g}\right)\right)
\end{aligned}
$$

where $F=d A$,

$$
V_{g}=\frac{V}{\sqrt{-g(V, V)}}, \quad \widetilde{V}_{g}=g\left(V_{g},-\right)
$$

\footnotetext{
${ }^{4}$ Since small variations in the metric $g$ do not change the timelike nature of $V$, its Gateaux derivative with respect to $g$ is zero.
} 
and the subscripts $g$ indicate explicit dependence on the metric. Again variation with respect to $A$ gives the Maxwell equation (8) where $G$ is given by (9). Thus

$$
\begin{aligned}
G=Z_{g}^{\mathrm{de}}\left(i_{V_{g}} F\right) & \wedge \widetilde{V}_{g}+Z_{g}^{\mathrm{db}}\left(i_{V_{g}} \star F\right) \wedge \widetilde{V}_{g} \\
-\star & \left(Z_{g}^{\mathrm{he}}\left(i_{V_{g}} F\right) \wedge \widetilde{V}_{g}\right)-\star\left(Z_{g}^{\mathrm{hb}}\left(i_{V_{g}} \star F\right) \wedge \widetilde{V}_{g}\right)
\end{aligned}
$$

where

$$
\begin{array}{ccc}
Z_{g}^{\mathrm{de}}=\frac{1}{2} \pi_{g} \circ\left(Z^{\mathrm{de}}+\left(Z^{\mathrm{de}}\right)^{\dagger}\right) \circ \pi_{g}, & Z_{g}^{\mathrm{db}}=\frac{1}{2} \pi_{g} \circ\left(Z^{\mathrm{db}}-\left(Z^{\mathrm{he}}\right)^{\dagger}\right) \circ \pi_{g}, \\
Z_{g}^{\mathrm{he}}=\frac{1}{2} \pi_{g} \circ\left(Z^{\mathrm{he}}-\left(Z^{\mathrm{db}}\right)^{\dagger}\right) \circ \pi_{g}, & Z_{g}^{\mathrm{hb}}=\frac{1}{2} \pi_{g} \circ\left(Z^{\mathrm{hb}}+\left(Z^{\mathrm{hb}}\right)^{\dagger}\right) \circ \pi_{g}, \\
\pi_{g}=\mathrm{Id}_{4}+\widetilde{V}_{g} \otimes V_{g} \quad \text { and } & \alpha \wedge \star Z_{g}^{\mathrm{I}}(\beta)=\beta \wedge \star\left(Z_{g}^{\mathrm{I}}\right)^{\dagger}(\alpha)
\end{array}
$$

for $Z_{g}^{\mathrm{I}} \in\left\{Z_{g}^{\mathrm{de}}, Z_{g}^{\mathrm{db}}, Z_{g}^{\mathrm{he}}, Z_{g}^{\mathrm{hb}}\right\}$ and $\alpha, \beta \in \Gamma \Lambda^{1} M$. This implies $Z_{g}^{\mathrm{I}}$ is spatial with respect to $V_{g}$, i.e. $Z_{g}^{\mathrm{I}}\left(\widetilde{V}_{g}\right)=0$ and $i_{V_{g}} Z_{g}^{\mathrm{I}}(\alpha)=0$ for all $\alpha \in \Gamma \Lambda^{1} M$. Since $\pi_{g}(\alpha) \wedge \widetilde{V}_{g}=\alpha \wedge \widetilde{V}_{g}$ and $\pi_{g}\left(i_{V_{g}} \gamma\right)=i_{V_{g}} \gamma$ for all $\alpha \in \Gamma \Lambda^{1} M$ and $\gamma \in \Gamma \Lambda^{2} M$ then $Z_{g}^{\text {de }}$ may be replaced by $\frac{1}{2}\left(Z^{\text {de }}+\right.$ $\left.\left(Z^{\mathrm{de}}\right)^{\dagger}\right)$ in $G$. Similarly $Z_{g}^{\mathrm{db}}$ may be replaced by $\frac{1}{2}\left(Z^{\mathrm{db}}-\left(Z^{\mathrm{he}}\right)^{\dagger}\right), Z_{g}^{\text {he }}$ by $\frac{1}{2}\left(Z^{\text {he }}-\left(Z^{\mathrm{db}}\right)^{\dagger}\right)$ and $Z_{g}^{\mathrm{hb}}$ by $\frac{1}{2}\left(Z^{\mathrm{hb}}+\left(Z^{\mathrm{hb}}\right)^{\dagger}\right)$. This expresses $G$ more simply in terms of the constitutive tensors in the action top form (12). Furthermore, after some rearrangement, one finds that $\frac{1}{2} F \wedge \star G=\Lambda^{\mathrm{m}},($ cf [3] $)$.

To facilitate the presentation below, it proves useful to relabel tensors in the arguments of $\Lambda^{\mathrm{m}}$ as

$$
\mathcal{Z}_{0}=g, \quad \mathcal{Z}_{A}=Z_{A}, \quad \mathcal{Z}_{N+2 B-1}=\zeta_{B} \quad \text { and } \quad \mathcal{Z}_{N+2 B}=d \zeta_{B}
$$

for $A=1, \ldots, N$ and $B=1, \ldots, Q$, so that

$$
\Lambda^{\mathbf{m}}\left(\mathcal{Z}_{0}, \ldots \mathcal{Z}_{N+2 Q}\right) \in \Gamma \Lambda^{n} M
$$

The range $C=0, \ldots, N+2 Q$ will be used to index the $\mathcal{Z}_{C}$.

In the following tensors of the form $\Psi=\Omega \otimes \Phi \in \Gamma\left(\Lambda^{n} M \otimes \otimes^{\bar{s}} M\right)$ where $\Omega \in \Gamma \Lambda^{n} M$ and $\Phi \in \Gamma \otimes^{\bar{s}} M$ arise naturally by "differentiating" $\Lambda^{\mathbf{m}}$ with respect to one of its arguments. One may contract such a tensor with $Y \in \Gamma \otimes^{s} M$ to isolate $\Omega$

$$
\Gamma\left(\Lambda^{n} M \otimes \otimes^{\bar{s}} M\right) \times \Gamma \otimes^{s} M \rightarrow \Gamma \Lambda^{n} M, \quad(\Psi, Y) \mapsto \Psi \vdots Y
$$

according to the rule

$$
(\Omega \otimes \Phi): Y=(\Phi: Y) \Omega
$$

where $\Omega \in \Gamma \Lambda^{n} M$ and $\Phi \in \Gamma \otimes^{\bar{s}} M$ and $Y \in \Gamma \otimes^{s} M$

\subsection{Variational Derivatives}

Using the indexing notation (16) and (17), the Gateaux derivative $\frac{\Delta \Lambda^{\mathbf{m}}}{\Delta \mathcal{Z}_{C}} \in \Gamma\left(\Lambda^{n} M \otimes\right.$ $\left.\otimes^{\overline{s_{C}}} M\right)$ of $\Lambda^{\mathrm{m}}$ with respect to $\mathcal{Z}_{C} \in \Gamma \otimes^{\boldsymbol{s}_{C}} M$ is defined so that

$$
\frac{\Delta \Lambda^{\mathbf{m}}}{\Delta \mathcal{Z}_{C}}: Y=\left.\frac{d}{d \varepsilon}\right|_{\varepsilon=0} \Lambda^{\mathbf{m}}\left(\mathcal{Z}_{0}, \ldots, \mathcal{Z}_{C-1}, \mathcal{Z}_{C}+\varepsilon Y, \mathcal{Z}_{C+1}, \ldots, \mathcal{Z}_{N+2 Q}\right)
$$


for all $Y \in \Gamma \otimes^{s_{C}} M$. An example in a local frame is given in appendix B,

By contrast, for $C=N+2 B-1$ then $\mathcal{Z}_{N+2 B-1}=\zeta_{B} \in \Gamma \Lambda^{p_{B}} M$ i.e. $\zeta_{B} \in \Gamma \otimes^{[\mathbb{F}, \ldots, \mathbb{F}]}$ (with a list of length $p_{B}$ ) the Gateaux derivative $\frac{\partial \Lambda^{\mathbf{m}}}{\partial \zeta_{B}} \in \Gamma \Lambda^{n-p_{B}} M$ is defined so that

$$
\begin{aligned}
\alpha \wedge \frac{\partial \Lambda^{\mathbf{m}}}{\partial \zeta_{B}} & =\left.\frac{d}{d \varepsilon}\right|_{\varepsilon=0} \Lambda^{\mathbf{m}}\left(g, Z_{1}, \ldots, Z_{N}, \zeta_{1}, d \zeta_{1}, \ldots, \zeta_{B}+\varepsilon \alpha, d \zeta_{B}, \ldots, \zeta_{Q}, d \zeta_{Q}\right) \\
& =\frac{\Delta \Lambda^{\mathbf{m}}}{\Delta \zeta_{B}}: \alpha
\end{aligned}
$$

for all $\alpha \in \Gamma \Lambda^{p_{B}} M$. The derivative $\frac{\partial \Lambda^{\mathbf{m}}}{\partial \zeta_{B}} \in \Gamma \Lambda^{n-p_{B}} M$ may be related to the derivative $\frac{\Delta \Lambda^{\mathbf{m}}}{\Delta \zeta_{B}} \in \Gamma\left(\Lambda^{n} M \otimes \otimes^{[\mathbb{V}, \ldots, \mathbb{V}]}\right)$ since one can identify $\Gamma \Lambda^{n-p_{B}} M$ and $\left\{\Omega \otimes \mathcal{V} \in \Gamma\left(\Lambda^{n} M \otimes\right.\right.$ $\left.\otimes^{[\mathbb{V}, \ldots, \mathbb{V}]}\right) \mid \mathcal{V}$ antisymmetric $\}$. The correspondence follows from the relation:

$$
(\Omega \otimes \mathcal{V}) \vdots \alpha=\alpha \wedge i_{\mathcal{V}} \Omega
$$

for any $\alpha \in \Gamma \Lambda^{p} M$. Here a general antisymmetric tensor $\mathcal{V} \in \Gamma \otimes^{[\mathbb{V}, \ldots, \mathbb{V}]} M$ can be written

$$
\begin{aligned}
\mathcal{V} & =\sum_{I_{1}<\ldots<I_{p}} \mathcal{V}^{I_{1} \cdots I_{p}} X_{I_{1}} \wedge \cdots \wedge X_{I_{p}} \\
& =\frac{1}{p !} \sum_{I_{1}<\ldots<I_{p}} \mathcal{V}^{I_{1} \cdots I_{p}} \sum_{\sigma \in S_{p}} \epsilon(\sigma) X_{\sigma\left(I_{1}\right)} \otimes \cdots \otimes X_{\sigma\left(I_{p}\right)}
\end{aligned}
$$

and the internal contraction operator $i_{\mathcal{V}}$ with respect to $\mathcal{V}$ is defined by:

$$
i_{\mathcal{V}}=\frac{1}{p !} \sum_{I_{1}<\ldots<I_{p}} \mathcal{V}^{I_{1} \cdots I_{p}} i_{X_{I_{p}}} \cdots i_{X_{I_{1}}}
$$

so that for $\alpha \in \Gamma \Lambda^{p} M$

$$
\alpha: \mathcal{V}=i_{\mathcal{V}} \alpha
$$

The proofs of (21) and (24) are given in lemmas 7 and 6 respectively in appendix A.

Likewise if $C=N+2 B$ then $\mathcal{Z}_{N+2 B}=d \zeta_{B} \in \Gamma \Lambda^{p_{B}+1} M$ and $\frac{\partial \Lambda^{\mathbf{m}}}{\partial\left(d \zeta_{B}\right)} \in \Gamma \Lambda^{n-p_{B}-1} M$ is defined by

$$
\begin{aligned}
\beta \wedge \frac{\partial \Lambda^{\mathbf{m}}}{\partial\left(d \zeta_{B}\right)} & =\left.\frac{d}{d \varepsilon}\right|_{\varepsilon=0} \Lambda^{\mathbf{m}}\left(g, Z_{1}, \ldots, Z_{N}, \zeta_{1}, d \zeta_{1}, \ldots, \zeta_{B}, d \zeta_{B}+\varepsilon \beta, \ldots, \zeta_{Q}, d \zeta_{Q}\right) \\
& =\frac{\Delta \Lambda^{\mathbf{m}}}{\Delta\left(d \zeta_{B}\right)} \vdots \beta
\end{aligned}
$$

for all $\beta \in \Gamma \Lambda^{p_{B}+1} M$.

The variational derivative $\frac{\delta \Lambda^{\mathrm{m}}}{\delta \zeta_{B}}$ of $\Lambda^{\mathrm{m}}$ with respect to $\zeta_{B} \in \Gamma \Lambda^{p_{B}} M$ is defined by

$$
\begin{aligned}
\int_{M} \beta \wedge \frac{\delta \Lambda^{\mathbf{m}}}{\delta \zeta_{B}}=\left.\frac{d}{d \varepsilon}\right|_{\varepsilon=0} \int_{M} \Lambda^{\mathbf{m}}\left(g, Z_{1}, \ldots, Z_{N}, \zeta_{1}, d \zeta_{1}, \ldots\right. \\
\left.\zeta_{B}+\varepsilon \beta, d \zeta_{B}+\varepsilon d \beta, \ldots, \zeta_{Q}, d \zeta_{Q}\right)
\end{aligned}
$$


for all $\beta \in \Gamma \Lambda^{p_{B}} M$ with compact support. Hence

$$
\frac{\delta \Lambda^{\mathbf{m}}}{\delta \zeta_{B}}=\frac{\partial \Lambda^{\mathbf{m}}}{\partial \zeta_{B}}+(-1)^{p_{B}+1} d\left(\frac{\partial \Lambda^{\mathbf{m}}}{\partial\left(d \zeta_{B}\right)}\right)
$$

follows from lemma 8 in appendix $\mathrm{A}$.

A $p$-form $\zeta_{B}$ is said to be "on $\Lambda^{\mathrm{m}}$-shell" if

$$
\frac{\delta \Lambda^{\mathbf{m}}}{\delta \zeta_{B}}=0
$$

In this situation

$$
\frac{\partial \Lambda^{\mathbf{m}}}{\partial \zeta_{B}}=(-1)^{p_{B}} d\left(\frac{\partial \Lambda^{\mathbf{m}}}{\partial\left(d \zeta_{B}\right)}\right)
$$

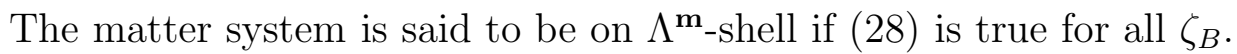

\subsection{Diffeomorphism invariance}

Given a local diffeomorphism $\phi: U_{M} \rightarrow U_{M}^{\prime}$ where $U_{M}, U_{M}^{\prime} \subset M$ then the two maps $\phi_{\star}$ and $\phi^{-1 \star}$ induce the map $\hat{\phi}: \otimes^{s} U_{M} \rightarrow \otimes^{s} U_{M}^{\prime}$ on tensors. The action top-form $\Lambda^{\mathbf{m}}\left(\mathcal{Z}_{0}, \ldots \mathcal{Z}_{N+2 Q}\right)$ is (locally) diffeomorphism invariant if

$$
\hat{\phi}\left(\Lambda^{\mathbf{m}}\left(\mathcal{Z}_{0}, \mathcal{Z}_{1}, \ldots, \mathcal{Z}_{N+2 Q}\right)\right)=\Lambda^{\mathbf{m}}\left(\hat{\phi}\left(\mathcal{Z}_{0}\right), \hat{\phi}\left(\mathcal{Z}_{1}\right), \ldots, \hat{\phi}\left(\mathcal{Z}_{N+2 Q}\right)\right)
$$

for all local diffeomorphism $\phi: U_{M} \rightarrow U_{M}^{\prime}$.

Lemma 1. If $\Lambda^{\mathbf{m}}$ is diffeomorphism invariant then for all vector fields $v \in \Gamma T M$

$$
\mathcal{L}_{v}\left(\Lambda^{\mathbf{m}}\left(\mathcal{Z}_{0}, \mathcal{Z}_{1}, \ldots, \mathcal{Z}_{N+2 Q}\right)\right)=\sum_{C=0}^{N+2 Q} \frac{\Delta \Lambda^{\mathbf{m}}}{\Delta \mathcal{Z}_{C}}: \mathcal{L}_{v} \mathcal{Z}_{C}
$$

which may be written

$$
\begin{aligned}
& \mathcal{L}_{v}\left(\Lambda^{\mathbf{m}}\left(g, Z_{1}, \ldots, Z_{N}, \zeta_{1}, d \zeta_{1}, \ldots, \zeta_{Q}, d \zeta_{Q}\right)\right)= \\
& \frac{\Delta \Lambda^{\mathbf{m}}}{\Delta g}: \mathcal{L}_{v} g+\sum_{A=1}^{N} \frac{\Delta \Lambda^{\mathbf{m}}}{\Delta Z_{A}}: \mathcal{L}_{v} Z_{A}+\sum_{B=1}^{Q} \mathcal{L}_{v} \zeta_{B} \wedge \frac{\partial \Lambda^{\mathbf{m}}}{\partial \zeta_{B}}+\sum_{B=1}^{Q} \mathcal{L}_{v}\left(d \zeta_{B}\right) \wedge \frac{\partial \Lambda^{\mathbf{m}}}{\partial\left(d \zeta_{B}\right)}
\end{aligned}
$$

Proof. Let $\phi_{\varepsilon}$ be the one parameter family of diffeomorphisms generated by $v$. From (30)

$$
\begin{aligned}
\mathcal{L}_{v} \Lambda^{\mathbf{m}}\left(\mathcal{Z}_{0}, \ldots, \mathcal{Z}_{N+2 Q}\right) & =\left.\frac{d}{d \varepsilon}\right|_{0} \hat{\phi}_{\varepsilon}\left(\Lambda^{\mathbf{m}}\left(\mathcal{Z}_{0}, \ldots, \mathcal{Z}_{N+2 Q}\right)\right) \\
& =\left.\frac{d}{d \varepsilon}\right|_{0} \Lambda^{\mathbf{m}}\left(\hat{\phi}_{\varepsilon}\left(\mathcal{Z}_{0}\right), \ldots, \hat{\phi}_{\varepsilon}\left(\mathcal{Z}_{N+2 Q}\right)\right) \\
& =\left.\sum_{C=0}^{N+2 Q} \frac{d}{d \varepsilon}\right|_{0} \Lambda^{\mathbf{m}}\left(\mathcal{Z}_{0}, \ldots, \hat{\phi}_{\varepsilon}\left(\mathcal{Z}_{C}\right), \ldots, \mathcal{Z}_{N+2 Q}\right) \\
& =\left.\sum_{C=0}^{N+2 Q} \frac{d}{d \varepsilon}\right|_{0} \Lambda^{\mathbf{m}}\left(\mathcal{Z}_{0}, \ldots, \mathcal{Z}_{C}+\varepsilon \mathcal{L}_{v} \mathcal{Z}_{C}, \ldots, \mathcal{Z}_{N+2 Q}\right) \\
& =\sum_{C=0}^{N+2 Q} \frac{\Delta \Lambda^{\mathbf{m}}}{\Delta \mathcal{Z}_{C}}: \mathcal{L}_{v} \mathcal{Z}_{C}
\end{aligned}
$$

Then (32) follows from (20) and (25). 
Since $\mathcal{L}_{v} \zeta_{B}=i_{v} d \zeta_{B}+d i_{v} \zeta_{B}$, the top-forms $\mathcal{L}_{v} \zeta_{B} \wedge \frac{\partial \Lambda^{\mathbf{m}}}{\partial \zeta_{B}}$ and $\mathcal{L}_{v}\left(d \zeta_{B}\right) \wedge \frac{\partial \Lambda^{\mathbf{m}}}{\partial\left(d \zeta_{B}\right)}$ in (31) can be expressed as

$$
\mathcal{L}_{v} \zeta_{B} \wedge \frac{\partial \Lambda^{\mathbf{m}}}{\partial \zeta_{B}}=d\left(i_{v} \zeta_{B} \wedge \frac{\partial \Lambda^{\mathbf{m}}}{\partial \zeta_{B}}\right)+(-1)^{p_{B}} i_{v} \zeta_{B} \wedge d \frac{\partial \Lambda^{\mathbf{m}}}{\partial \zeta_{B}}+i_{v} d \zeta_{B} \wedge \frac{\partial \Lambda^{\mathbf{m}}}{\partial \zeta_{B}}
$$

and

$$
\mathcal{L}_{v}\left(d \zeta_{B}\right) \wedge \frac{\partial \Lambda^{\mathbf{m}}}{\partial\left(d \zeta_{B}\right)}=d\left(i_{v} d \zeta_{B} \wedge \frac{\partial \Lambda^{\mathbf{m}}}{\partial\left(d \zeta_{B}\right)}\right)-(-1)^{p_{B}} i_{v} d \zeta_{B} \wedge d \frac{\partial \Lambda^{\mathbf{m}}}{\partial\left(d \zeta_{B}\right)}
$$

where $\zeta_{B} \in \Gamma \Lambda^{p_{B}} M$. See lemma 9 in the appendix $\mathrm{A}$.

By analogy with (33) and (34), the top-forms $\frac{\Delta \Lambda^{\mathbf{m}}}{\Delta \mathcal{Z}_{C}}: \mathcal{L}_{v} \mathcal{Z}_{C}$ in (31) may be written

$$
\frac{\Delta \Lambda^{\mathrm{m}}}{\Delta \mathcal{Z}_{C}}: \mathcal{L}_{v} \mathcal{Z}_{C}=d\left(\mathcal{A}_{v}\left(\frac{\Delta \Lambda^{\mathrm{m}}}{\Delta \mathcal{Z}_{C}}, \mathcal{Z}_{C}\right)\right)+\mathcal{B}_{v}\left(\frac{\Delta \Lambda^{\mathrm{m}}}{\Delta \mathcal{Z}_{C}}, \mathcal{Z}_{C}\right)
$$

where the maps

$$
\mathcal{A}: \Gamma T M \times \Gamma\left(\Lambda^{n} M \otimes \otimes^{\bar{s}} M\right) \times \Gamma \otimes^{s} M \rightarrow \Gamma \Lambda^{n-1} M, \quad(v, \Psi, Z) \mapsto \mathcal{A}_{v}(\Psi, Z)
$$

and

$$
\mathcal{B}: \Gamma T M \times \Gamma\left(\Lambda^{n} M \otimes \otimes^{\bar{s}} M\right) \times \Gamma \otimes^{s} M \rightarrow \Gamma \Lambda^{n} M, \quad(v, \Psi, Z) \mapsto \mathcal{B}_{v}(\Psi, Z)
$$

are 'f'-linear in $v$ and defined inductively as follows: For 0-forms, $f \in \Gamma \otimes^{[]} M$

$$
\mathcal{A}_{v}(\Omega, f)=0 \quad \text { and } \quad \mathcal{B}_{v}(\Omega, f)=v(f) \Omega
$$

For 1-forms $\alpha \in \Gamma \otimes^{[\mathbb{F}]} M$

$$
\begin{aligned}
& \mathcal{A}_{v}(\Omega \otimes u, \alpha)=\alpha(v) i_{u} \Omega \\
& \text { and } \quad \mathcal{B}_{v}(\Omega \otimes u, \alpha)=(-1)^{n+1} i_{u} \Omega \wedge i_{v} d \alpha-d i_{u} \Omega \wedge i_{v} \alpha
\end{aligned}
$$

For vectors $u \in \Gamma \otimes^{[\mathbb{V}]} M$

$$
\begin{aligned}
& \mathcal{A}_{v}(\Omega \otimes \alpha, u)=-\alpha(v) i_{u} \Omega \\
& \text { and } \quad \mathcal{B}_{v}(\Omega \otimes \alpha, u)=v(\alpha(u)) \Omega-i_{u} \Omega \wedge i_{v} d \alpha+(-1)^{n} d i_{u} \Omega \wedge i_{v} \alpha
\end{aligned}
$$

For tensors $\Phi_{1} \in \Gamma \otimes^{\bar{s}} M, \Phi_{2} \in \Gamma \otimes^{\bar{t}} M, Z_{1} \in \Gamma \otimes^{s} M, Z_{2} \in \Gamma \otimes^{t} M$ and $\Omega \in \Gamma \Lambda^{n} M$, define the Leibnitz rules:

$$
\begin{aligned}
& \mathcal{A}_{v}\left(\Omega \otimes \Phi_{1} \otimes \Phi_{2}, Z_{1} \otimes Z_{2}\right) \\
& \quad=\mathcal{A}_{v}\left(\left(\Phi_{1}: Z_{1}\right) \Omega \otimes \Phi_{2}, Z_{2}\right)+\mathcal{A}_{v}\left(\left(\Phi_{2}: Z_{2}\right) \Omega \otimes \Phi_{1}, Z_{1}\right) \\
& \mathcal{B}_{v}\left(\Omega \otimes \Phi_{1} \otimes \Phi_{2}, Z_{1} \otimes Z_{2}\right) \\
& \quad=\mathcal{B}_{v}\left(\left(\Phi_{1}: Z_{1}\right) \Omega \otimes \Phi_{2}, Z_{2}\right)+\mathcal{B}_{v}\left(\left(\Phi_{2}: Z_{2}\right) \Omega \otimes \Phi_{1}, Z_{1}\right)
\end{aligned}
$$

The proof that (35) follows from lemma 10 given in appendix $\mathrm{A}$. The proof that (33) and (34) are consistent with (35) is given in lemma 11 appendix A. 
If $\Lambda^{\mathbf{m}}$ is diffeomorphism invariant then from (31) and (351):

$$
\begin{aligned}
d i_{v} \Lambda^{\mathbf{m}} & =\mathcal{L}_{v} \Lambda^{\mathbf{m}}=\sum_{C=0}^{N+2 Q} \frac{\Delta \Lambda^{\mathbf{m}}}{\Delta \mathcal{Z}_{C}}: \mathcal{L}_{v} \mathcal{Z}_{C} \\
& =d\left(\sum_{C=0}^{N+2 Q} \mathcal{A}_{v}\left(\frac{\Delta \Lambda^{\mathbf{m}}}{\Delta \mathcal{Z}_{C}}, \mathcal{Z}_{C}\right)\right)+\sum_{C=0}^{N+2 Q} \mathcal{B}_{v}\left(\frac{\Delta \Lambda^{\mathbf{m}}}{\Delta \mathcal{Z}_{C}}, \mathcal{Z}_{C}\right)
\end{aligned}
$$

hence applying lemma 5, appendix $\mathrm{A}$ gives

$$
i_{v} \Lambda^{\mathbf{m}}=\sum_{C=0}^{N+2 Q} \mathcal{A}_{v}\left(\frac{\Delta \Lambda^{\mathbf{m}}}{\Delta \mathcal{Z}_{C}}, \mathcal{Z}_{C}\right)
$$

and

$$
\sum_{C=0}^{N+2 Q} \mathcal{B}_{v}\left(\frac{\Delta \Lambda^{\mathbf{m}}}{\Delta \mathcal{Z}_{C}}, \mathcal{Z}_{C}\right)=0
$$

Using (33) and (34), these may be written as

$$
\begin{aligned}
\mathcal{A}_{v}\left(\frac{\Delta \Lambda^{\mathbf{m}}}{\Delta g}, g\right)=i_{v} \Lambda^{\mathbf{m}} & -\sum_{A=1}^{N} \mathcal{A}_{v}\left(\frac{\Delta \Lambda^{\mathbf{m}}}{\Delta Z_{A}}, Z_{A}\right) \\
& -\sum_{B=1}^{Q}\left(i_{v} \zeta_{B} \wedge \frac{\partial \Lambda^{\mathbf{m}}}{\partial \zeta_{B}}+i_{v} d \zeta_{B} \wedge \frac{\partial \Lambda^{\mathbf{m}}}{\partial\left(d \zeta_{B}\right)}\right)
\end{aligned}
$$

and

$$
\begin{aligned}
\mathcal{B}_{v}\left(\frac{\Delta \Lambda^{\mathbf{m}}}{\Delta g}, g\right)=-\sum_{A=1}^{N} & \mathcal{B}_{v}\left(\frac{\Delta \Lambda^{\mathbf{m}}}{\Delta Z_{A}}, Z_{A}\right)-\sum_{B=1}^{Q}\left((-1)^{p_{B}} i_{v} \zeta_{B} \wedge d \frac{\partial \Lambda^{\mathbf{m}}}{\partial \zeta_{B}}\right. \\
+i_{v} d \zeta_{B} & \left.\wedge \frac{\partial \Lambda^{\mathbf{m}}}{\partial \zeta_{B}}+(-1)^{p_{B}+1} i_{v} d \zeta_{B} \wedge d \frac{\partial \Lambda^{\mathbf{m}}}{\partial\left(d \zeta_{B}\right)}\right)
\end{aligned}
$$

When the system is on $\Lambda^{\mathbf{m}}$-shell (43) may be written

$$
\begin{aligned}
\mathcal{A}_{v}\left(\frac{\Delta \Lambda^{\mathbf{m}}}{\Delta g}, g\right)=i_{v} \Lambda^{\mathbf{m}}- & \sum_{B=1}^{Q} \mathcal{L}_{v} \zeta_{B} \wedge \frac{\partial \Lambda^{\mathbf{m}}}{\partial\left(d \zeta_{B}\right)} \\
& +d\left(\sum_{B=1}^{Q} i_{v} \zeta_{B} \wedge \frac{\partial \Lambda^{\mathbf{m}}}{\partial\left(d \zeta_{B}\right)}\right)-\sum_{A=1}^{N} \mathcal{A}_{v}\left(\frac{\Delta \Lambda^{\mathbf{m}}}{\Delta Z_{A}}, Z_{A}\right)
\end{aligned}
$$

whereas (44) reduces to

$$
\mathcal{B}_{v}\left(\frac{\Delta \Lambda^{\mathbf{m}}}{\Delta g}, g\right)=-\sum_{A=1}^{N} \mathcal{B}_{v}\left(\frac{\Delta \Lambda^{\mathbf{m}}}{\Delta Z_{A}}, Z_{A}\right)
$$

See lemma 12 appendix A. Relations (43)-(46) play a pivotal role in the arguments below. In particular they enable one identify terms which may be associated with the quantities derived historically with Noether, Belinfante and Rosenfeld. 


\section{Currents and Conservation laws}

Many quantities in physics owe their raison-d'etre to the existence of conserved quantities that do not change with time in a dynamical system. Thus notions of energy, momentum and angular momentum arose from the analysis of Newtonian particle dynamics. With the introduction of fields and the development of continuum mechanics it became natural to incorporate such concepts into continuous dynamical systems and their unification into a "stress-energy-momentum" complex offered an attractive objective. However, as is well known such a unification is not unique and the indiscriminate use of the term "stressenergy-momentum" tensor has led to unnecessary confusion when discussing forces and torques produced by fields in media.

In this section a number of technical issues are addressed that inter-relate these physical concepts. They include the role played by different aspects of (multi-)linearity needed for a general definition of stress over curved surfaces in space, the role of Killing symmetry needed to establish the notions of energy and momentum and the role of algebraic symmetry of maps and associated tensors or tensor-densities in their conservation. Using the variational framework established in the previous section it is possible to correlate these aspects with the parts played by the presence or absence of background matter fields and background gravitation in their implementation.

Since some of the traditional arguments for the construction of a symmetric stressenergy-momentum tensor in Minkowski spacetime are spurious (even in the absence of background matter fields) it is useful to begin the discussion with the Noether current associated with $\Lambda^{\mathrm{m}}$ in a general background using the Lie-derivative. This leads naturally to conservation laws in the presence of background symmetries. In this manner it is also straightforward to extricate the role played by $f$-linearity in establishing a proper tensor description of stress.

\subsection{Noether and Belinfante-Rosenfeld currents}

For the restricted class of actions considered in this article we define the Noether $(n-1)$ form current associated with $\Lambda^{\mathrm{m}}$ by

$$
\mathcal{N}_{v}=i_{v} \Lambda^{\mathbf{m}}-\sum_{B=1}^{Q} \mathcal{L}_{v} \zeta_{B} \wedge \frac{\partial \Lambda^{\mathbf{m}}}{\partial\left(d \zeta_{B}\right)}
$$

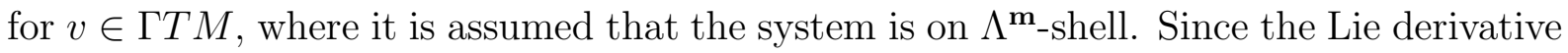
$\mathcal{L}_{v}$ is not $f$-linear in $v$ neither is $\mathcal{N}_{v}$ and for $v=v^{a} \partial_{a}$, lemma 13 in appendix A gives

$$
\mathcal{N}_{v}=v^{a} \mathcal{N}_{\partial_{a}}+\sum_{B=1}^{Q} d v^{a} \wedge i_{\partial_{a}} \zeta_{B} \wedge \frac{\partial \Lambda^{\mathbf{m}}}{\partial\left(d \zeta_{B}\right)}
$$

It is however $\mathbb{R}$-linear in $v$ (where $v^{a}$ are constants). For a chosen nowhere vanishing $\Omega \in \Gamma \Lambda^{n} M$ one may define the map $\mathcal{T}^{\mathcal{N}}: \Gamma T M \rightarrow \Gamma T M$ by

$$
\mathcal{N}_{v}=i_{\mathcal{T N}(v)} \Omega
$$

In a coordinate system $\left(x^{1}, \ldots, x^{n}\right)$ with $\Omega=d x^{1} \wedge \cdots \wedge d x^{n}$ and $\Lambda^{\mathbf{m}}=\mathcal{S} \Omega$ then $\mathcal{T}^{\mathcal{N}}$ has component maps

$$
\mathcal{T}^{\mathcal{N} a}{ }_{b}=d x^{a}\left(\mathcal{T}^{\mathcal{N}}\left(\partial_{b}\right)\right)
$$


which from lemma 15 in appendix $\mathrm{A}$ gives

$$
d x^{a} \wedge \mathcal{N}_{\partial_{b}}=\mathcal{T}^{\mathcal{N} a}{ }_{b} \Omega \quad \text { and } \quad \mathcal{T}^{\mathcal{N} a}{ }_{b}=i_{\partial_{n}} \cdots i_{\partial_{1}}\left(d x^{a} \wedge \mathcal{N}_{\partial_{b}}\right)
$$

Lemma 27 in appendix $\mathrm{A}$ then gives

$$
\mathcal{T}^{\mathcal{N} a}{ }_{b}=\delta_{b}^{a} \mathcal{S}-\sum_{B=1}^{Q} \sum_{I_{1}<\cdots<I_{p_{B}}} \partial_{b}\left(\zeta_{B I}\right) \frac{\partial \mathcal{S}}{\partial\left(\partial_{a} \zeta_{B I}\right)}
$$

where

$$
\zeta_{B}=\sum_{I_{1}<\cdots<I_{p_{B}}} \zeta_{B I} d x^{I_{1}} \wedge \cdots \wedge d x^{I_{p_{B}}}
$$

Since $\mathcal{T}^{\mathcal{N}}$ is not $f$-linear, i.e. $\mathcal{T}^{\mathcal{N}}(f v) \neq f \mathcal{T}^{\mathcal{N}}(v)$ in general, one must not confuse $\mathcal{T}^{\mathcal{N}}{ }_{b}{ }^{a}$ with the components of a tensor field. In fact using (48) and lemma 15 in the appendix for $v=v^{a} \partial_{a}$,

$$
\mathcal{T}^{\mathcal{N}}(v)=\left(\mathcal{T}^{\mathcal{N}}\right)^{a}{ }_{b} v^{b}+\left(\partial_{c} v^{b}\right) i_{\partial_{1}} \cdots i_{\partial_{n}}\left(\sum_{B=1}^{Q} d x^{a} \wedge d x^{c} \wedge i_{\partial_{b}} \zeta_{B} \wedge \frac{\partial \Lambda^{\mathbf{m}}}{\partial\left(d \zeta_{B}\right)}\right)
$$

However, it is not uncommon to refer to (152) as the components of the canonical stressenergy-momentum tensor associated with $\Lambda^{\mathrm{m}}$ in Minkowski spacetime [4, 11, 13. This arises since $\mathcal{T}^{\mathcal{N}}{ }_{b}{ }^{a} \rightarrow R^{a}{ }_{c} \mathcal{T}^{\mathcal{N}}{ }_{b}{ }^{c}$, under affine coordinate transformations of the form

$$
x^{a} \rightarrow y^{a}=R_{b}^{a} x^{b}+B^{a}
$$

where $\operatorname{det}(R)=1$ and $R_{b}^{a}, B^{a} \in \mathbb{R}$ are constants. This accounts for its widespread use in special relativity as formulated by Einstein and Minkowski in spacetime $\mathcal{M}$. Since $\mathcal{T}^{\mathcal{N}}$ is not tensorial with respect to arbitrary coordinate transformations its use for calculating stresses is restricted to planar surfaces in space. This follows from (53), since for any event $p \in \mathcal{M}$ on a non-planar spacelike 2-surface with normal field $w,\left.\mathcal{T}^{\mathcal{N}}(w)\right|_{p}$ will depend on the derivatives $\left.\left(\partial_{a} w^{b}\right)\right|_{p}$. Thus one cannot, in general, define Cauchy traction forces that must be independent of such derivatives [12].

The requirement that the concept of stress follows from a bona-fide tensor leads one to seek an $f$-linear map constructed from $\mathcal{N}_{v}$. Since $\mathcal{L}_{v}=d i_{v}+i_{v} d$, the $(n-1)$-form current

$$
\tau_{v}^{\mathcal{B}}=\mathcal{N}_{v}+d S_{v}
$$

where

$$
S_{v}=\sum_{B=1}^{Q} i_{v} \zeta_{B} \wedge \frac{\partial \Lambda^{\mathbf{m}}}{\partial\left(d \zeta_{B}\right)}
$$

is manifestly $f$-linear in $v$. One may refer to this as a Belinfante-Rosenfeld formula [5] although one may note that no metric on $M$ is necessary for its construction. It follows from (47) and (55) that

$$
\tau_{v}^{\mathcal{B}}=i_{v} \Lambda^{\mathbf{m}}-\sum_{B=1}^{Q}\left(i_{v} \zeta_{B} \wedge \frac{\partial \Lambda^{\mathbf{m}}}{\partial \zeta_{B}}+i_{v} d \zeta_{B} \wedge \frac{\partial \Lambda^{\mathbf{m}}}{\partial\left(d \zeta_{B}\right)}\right)
$$


which we refer to as the Belinfante-Rosenfeld stress-energy-momentum current associated with $\Lambda^{\mathbf{m}}$. From (43) one can also write (56) in terms of the maps $\mathcal{A}_{v}$ as

$$
\tau_{v}^{\mathcal{B}}=\mathcal{A}_{v}\left(\frac{\Delta \Lambda^{\mathrm{m}}}{\Delta g}, g\right)+\sum_{A=1}^{N} \mathcal{A}_{v}\left(\frac{\Delta \Lambda^{\mathrm{m}}}{\Delta Z_{A}}, Z_{A}\right)
$$

Again, for a chosen nowhere vanishing $\Omega \in \Gamma \Lambda^{n} M$ the map $\mathcal{T}^{\mathcal{B}} \in \Gamma \otimes^{[\mathbb{V}, \mathbb{F}]}$ defined $[5$

$$
\tau_{v}^{\mathcal{B}}=i_{\mathcal{T} \mathcal{B}(v)} \Omega
$$

is a density with respect to $\Omega$ with weight -1 .

Given a preferred metric $g$ and any map $\mathcal{T}: \Gamma T M \rightarrow \Gamma T M$ one may define the map $T: \Gamma T M \times \Gamma T M \rightarrow \Gamma \Lambda^{0} M$ by

$$
T(u, v)=g(\mathcal{T}(u), v)
$$

This enables one to discuss the algebraic symmetries of $T$. Such a map is said to be algebraically symmetric with respect to $g$ if

$$
T(u, v)=T(v, u)
$$

This implies (see lemma 14 in appendix A)

$$
\widetilde{v} \wedge i_{\mathcal{T}(u)} \Omega=\widetilde{u} \wedge i_{\mathcal{T}(v)} \Omega
$$

for all $u, v \in \Gamma T M$ any non-vanishing top-form $\Omega \in \Lambda^{n} M$. Thus for the maps $T^{\mathcal{N}}$ : $\Gamma T M \times \Gamma T M \rightarrow \Gamma \Lambda^{0} M$ and $T^{\mathcal{B}}: \Gamma T M \times \Gamma T M \rightarrow \Gamma \Lambda^{0} M$

$$
T^{\mathcal{N}}(u, v)=g\left(\mathcal{T}^{\mathcal{N}}(u), v\right) \quad \text { and } \quad T^{\mathcal{B}}(u, v)=g\left(\mathcal{T}^{\mathcal{B}}(u), v\right)
$$

Then $T^{\mathcal{N}}$ is symmetric if

$$
\widetilde{u} \wedge \mathcal{N}_{v}=\widetilde{v} \wedge \mathcal{N}_{u} \quad \text { for all } \quad u, v \in \Gamma T M
$$

and $T^{\mathcal{B}}$ is symmetric if

$$
\widetilde{u} \wedge \tau_{v}^{\mathcal{B}}=\widetilde{v} \wedge \tau_{u}^{\mathcal{B}} \quad \text { for all } \quad u, v \in \Gamma T M
$$

To illustrate these notions, consider the premetric formulation of electromagnetism [8, 6] on spacetime $\mathcal{M}$ where one starts with the action

$$
\Lambda^{\mathbf{m}}\left(Z_{1}, \ldots, Z_{N}, d A\right)=\frac{1}{2} F \wedge \mathcal{H}
$$

with $F=d A$ and $\mathcal{H}=\kappa\left(Z_{1}, \ldots, Z_{N}, F\right)$ linear in $F$. The Noether current is then

$$
\mathcal{N}_{v}=\frac{1}{2} F \wedge i_{v} \mathcal{H}-\frac{1}{2} i_{v} F \wedge \mathcal{H}+d i_{v} A \wedge \mathcal{H}
$$

\footnotetext{
${ }^{5}$ Some authors [11, 13] refer to $\mathcal{T}^{\mathcal{N}}$ as the canonical stress-energy-momentum tensor whereas others [8, 10, 7] refer to $\mathcal{T}^{\mathcal{B}}$ as the canonical stress-energy-momentum tensor. The appellations $\mathcal{T}^{\mathcal{N}}$ and $\mathcal{T}^{\mathcal{B}}$ eliminate this notational ambiguity.
} 
This is manifestly not $U(1)$ gauge invariant. However the Belinfante-Rosenfeld current (54)

$$
\tau_{v}^{\mathcal{B}}=\frac{1}{2} F \wedge i_{v} \mathcal{H}-\frac{1}{2} i_{v} F \wedge \mathcal{H}
$$

is $U(1)$ gauge invariant.

When a metric is prescribed as in the spacetime model (4) where

$$
\Lambda^{\mathrm{m}}\left(g, Z_{1}, \ldots, Z_{N}, d A\right)=\frac{1}{2} F \wedge \star G
$$

with $G=\chi\left(g, Z_{1}, \ldots, Z_{N}, F\right)$ linear in $F$, the Noether current is given by (65) with $\mathcal{H}=\star G$ and the Belinfante-Rosenfeld current is given by (66) with $\mathcal{H}=\star G$. In this case the Belinfante-Rosenfeld current

$$
\tau_{v}^{\mathcal{B}}=\frac{1}{2} F \wedge i_{v} \star G-\frac{1}{2} i_{v} F \wedge \star G
$$

gives rise via (58) and (59) to the algebraically non-symmetric Minkowski stress-energymomentum tensor [9] density $T^{\mathcal{B}}$. Examples (5) and (12) are particular cases of (67).

In general, neither $T^{\mathcal{N}}$ nor $T^{\mathcal{B}}$ possess the algebraic symmetry (159) due to the presence of background fields. In the particular case of the vacuum where $\mathcal{H}=\star F$, then $T^{\mathcal{B}}$ has algebraic symmetry, while $T^{\mathcal{N}}$ remains non-symmetric. In the absence of a preferred metric one cannot even define $T^{\mathcal{N}}$ or $T^{\mathcal{B}}$ from $\mathcal{T}^{\mathcal{N}}$ and $\mathcal{T}^{\mathcal{B}}$ respectively.

\subsection{Conservation Laws}

In the presence of Lie-symmetries of $g$ and $Z_{A}$, both the Noether and Belinfante-Rosenfeld stress-energy-momentum current give rise to conserved material quantities.

Theorem 2. If $K \in \Gamma T M$ is a Killing vector field, i.e. $\mathcal{L}_{K} g=0$, and in addition the background tensor fields $\{\boldsymbol{Z}\}$ satisfy the Lie-symmetry condition $\mathcal{L}_{K} Z_{A}=0$ for $A=$ $1, \ldots, N$, then both the Noether current and the Belinfante-Rosenfeld current are closed

$$
d \tau_{K}^{\mathcal{B}}=d \mathcal{N}_{K}=0
$$

Proof. Since $\mathcal{L}_{K} g=0$ and $\mathcal{L}_{K} Z_{A}=0$ then from (35)

$$
d\left(\mathcal{A}_{K}\left(\frac{\Delta \Lambda^{\mathbf{m}}}{\Delta g}, g\right)\right)+\mathcal{B}_{K}\left(\frac{\Delta \Lambda^{\mathbf{m}}}{\Delta g}, g\right)=0
$$

and

$$
d\left(\mathcal{A}_{K}\left(\frac{\Delta \Lambda^{\mathbf{m}}}{\Delta Z_{A}}, Z_{A}\right)\right)+\mathcal{B}_{K}\left(\frac{\Delta \Lambda^{\mathbf{m}}}{\Delta Z_{A}}, Z_{A}\right)=0
$$

for all $Z_{A}$. Thus from (57) and (46)

$$
\begin{aligned}
d \tau_{K}^{\mathcal{B}} & =d\left(\mathcal{A}_{K}\left(\frac{\Delta \Lambda^{\mathbf{m}}}{\Delta g}, g\right)\right)+\sum_{A=1}^{N} d\left(\mathcal{A}_{K}\left(\frac{\Delta \Lambda^{\mathbf{m}}}{\Delta Z_{A}}, Z_{A}\right)\right) \\
& =-\mathcal{B}_{K}\left(\frac{\Delta \Lambda^{\mathbf{m}}}{\Delta g}, g\right)-\sum_{A=1}^{N} \mathcal{B}_{K}\left(\frac{\Delta \Lambda^{\mathbf{m}}}{\Delta Z_{A}}, Z_{A}\right)=0
\end{aligned}
$$


In terms of the maps $\mathcal{T}^{\mathcal{N}}$ and $\mathcal{T}^{\mathcal{B}}$, using lemma 15 in appendix $\mathrm{A}$, (69) may be written in a general coordinate basis with $\Omega=d x^{1} \wedge \cdots \wedge d x^{n}$ as

$$
\partial_{a}\left(\left(\mathcal{T}^{\mathcal{B}}\right)^{a}{ }_{b} K^{b}\right)=0
$$

and from (53)

$$
\partial_{a}\left(\left(\mathcal{T}^{\mathcal{N}}\right)_{b}^{a} K^{b}+\left(\partial_{c} K^{b}\right) i_{\partial_{1}} \cdots i_{\partial_{n}}\left(\sum_{B=1}^{Q} d x^{a} \wedge d x^{c} \wedge i_{\partial_{b}} \zeta_{B} \wedge \frac{\partial \Lambda^{\mathbf{m}}}{\partial\left(d \zeta_{B}\right)}\right)\right)=0
$$

When $g$ is Lorentzian on spacetime, by Stoke's theorem both $\mathcal{T}^{\mathcal{B}}$ and $\mathcal{T}^{\mathcal{N}}$ yield conservation laws. An energy conservation law follows if $K$ is timelike, a linear momentum conservation law if there exist three independent spacelike translational Killing vectors and an angular momentum conservation law follows if there exists a basis of three independent spacelike Killing vectors generating spatial rotations. Such conservation laws make no reference to forces (stress) or torques (moments) and are valid in the presence of smooth non-dynamical background fields. However only $\mathcal{T}^{\mathcal{B}}$, being $f$-linear, deserves the appellation stress-energy-momentum tensor.

If $\Lambda^{\mathbf{m}}$ does not depend on a metric $g$ the requirement that $K$ is a Killing vector may be dropped, i.e. if $\Lambda^{\mathbf{m}}\left(Z_{1}, \ldots, Z_{N}, \zeta_{1}, d \zeta_{1}, \ldots, \zeta_{Q}, d \zeta_{Q}\right)$ is independent of $g$ and $\mathcal{L}_{V} Z_{A}=0$ for $A=1, \ldots, N$ and $V \in \Gamma T M$, then

$$
d \tau_{V}^{\mathcal{B}}=d \mathcal{N}_{V}=0
$$

However, in the absence of a metric, no physical concept of energy or momentum exists.

If $\mathcal{L}_{K} g=0$ but not all background fields $\{\boldsymbol{Z}\}$ are Lie-symmetric then a simple generalisation of theorem 2 yields

$$
\begin{aligned}
d \mathcal{N}_{K}=d \tau_{K}^{\mathcal{B}} & =\sum_{\left\{A \mid \mathcal{L}_{K} Z_{A} \neq 0\right\}}\left(d \mathcal{A}_{K}\left(\frac{\Delta \Lambda^{\mathbf{m}}}{\Delta Z_{A}}, Z_{A}\right)+\mathcal{B}_{K}\left(\frac{\Delta \Lambda^{\mathbf{m}}}{\Delta Z_{A}}, Z_{A}\right)\right) \\
& =\sum_{\left\{A \mid \mathcal{L}_{K} Z_{A} \neq 0\right\}} \frac{\Delta \Lambda^{\mathbf{m}}}{\Delta Z_{A}}: \mathcal{L}_{K} Z_{A}
\end{aligned}
$$

which in general is not equal to zero.

\subsection{Historical perspectives in Minkowski spacetime}

In the absence of background fields $\{\boldsymbol{Z}\}$ in Minkowski spacetime, $T^{\mathcal{B}}{ }_{a b}=T^{\mathcal{B}}{ }_{b a}$ and from (71), $\partial_{a}\left(T^{\mathcal{B} a}{ }_{b}\right)=0$. It follows that the "moment of $T^{\mathcal{B}}{ }_{\nu 0}$ " is conserved. Thus in inertial coordinates $\left(x^{0}, x^{1}, x^{2}, x^{3}\right)$ with

$$
M^{\mathcal{B}}{ }_{\mu \nu}=-\left(x_{\mu} T^{\mathcal{B}}{ }_{\nu 0}-x_{\nu} T^{\mathcal{B}}{ }_{\nu 0}\right)
$$

for $\mu, \nu=1,2,3$, vanishing at spatial infinity, one has from (71) with $K=x_{\mu} \partial_{\nu}-x_{\nu} \partial_{\mu}$

$$
\frac{d}{d x^{0}} \int_{\mathbb{R}^{3}} M^{\mathcal{B}}{ }_{\mu \nu} d x^{1} \wedge d x^{2} \wedge d x^{3}=0
$$


This is identified with the conservation of (orbital) angular momentum of a field system in $\mathbb{R}^{3}$. This result is a direct consequence of (71) which does not require the imposition of any algebraical symmetry.

More generally with the Killing vector fields, for $a, b=0,1,2,3$

$$
\mathcal{R}_{a b}=x_{a} \partial_{b}-x_{b} \partial_{a}
$$

and

$$
T^{\mathcal{B}}\left(R_{a b}\right)=\mathcal{M}^{\mathcal{B}}{ }_{a b}^{c} \partial_{c}
$$

where

$$
\mathcal{M}^{\mathcal{B}}{ }_{a b}{ }^{c}=T^{\mathcal{B} c}{ }_{a} x_{b}-T^{\mathcal{B} c}{ }_{b} x_{a}
$$

is the "moment of $T^{\mathcal{B} c}$ ", one has

$$
\partial_{c} \mathcal{M}^{\mathcal{B}}{ }_{a b}^{c}=0
$$

In fact (71) shows that (75) and (79) are valid in the presence of Lie-symmetric background fields satisfying $\mathcal{L}_{\mathcal{R}_{a b}} Z_{A}=0$ for all $A$ and for all $\mathcal{R}_{a b}$.

In Minkowski spacetime there exists a basis of 10 Killing vector fields generating the algebra of the Poincaré group. The nature of the algebraic symmetry of $T^{\mathcal{B}}{ }_{a b}$ depends on the Lie symmetry of the background fields. This is a consequence of the following lemma:

Lemma 3. In Minkowski spacetime, for fixed $a$ and $b$, let $\partial_{a}$ and $\partial_{b}$ be two commuting translational Killing vectors such that $\mathcal{L}_{\partial_{a}} Z_{A}=0$ and $\mathcal{L}_{\partial_{b}} Z_{A}=0$ for all $A$. Then $T^{\mathcal{B}}$ is algebraically partially symmetric if and only if $\tau_{\mathcal{R}_{a b}}$ is closed, i.e.

$$
T^{\mathcal{B}}{ }_{a b}=T^{\mathcal{B}}{ }_{b a} \quad \Leftrightarrow \quad d \tau_{R_{a b}}^{\mathcal{B}}=0
$$

Proof. Since $\mathcal{L}_{\partial_{a}} Z_{A}=0$ and $\mathcal{L}_{\partial_{b}} Z_{A}=0$ then $d \tau_{\partial_{a}}=0$ and $d \tau_{\partial_{b}}=0$. From $f$-linearity $\tau_{R_{a b}}^{\mathcal{B}}=x_{a} \tau_{\partial_{b}}^{\mathcal{B}}-x_{b} \tau_{\partial_{a}}^{\mathcal{B}}$ hence from (60)

$$
d \tau_{R_{a b}}^{\mathcal{B}}=d x_{a} \wedge \tau_{\partial_{b}}^{\mathcal{B}}-d x_{b} \wedge \tau_{\partial_{a}}^{\mathcal{B}}=\left(T_{a b}^{\mathcal{B}}-T_{b a}^{\mathcal{B}}\right) d x^{0} \wedge d x^{1} \wedge d x^{2} \wedge d x^{3}
$$

Hence (80).

Thus total (orbital) angular momentum conservation in Minkowski spacetime, generated by $\left\{\partial_{1}, \partial_{2}, \partial_{3}\right\}$, does not demand that $T^{\mathcal{B}}{ }_{a b}$ is fully algebraically symmetric for all $a, b$.

It is of interest to note that conservation of an $S O(3,1)$ Killing current can be obtained directly from the Noether quantities in the absence of background fields. It follows from (69) and (71) that for all Killing vector fields $\mathcal{R}_{a b}$ one has

$$
d \mathcal{N}_{\mathcal{R}_{a b}}=0
$$

and

$$
\partial_{c}\left(T^{\mathcal{N}}\left(R_{a b}\right)^{c}\right)=0
$$


where from (48)

$$
\mathcal{N}_{\mathcal{R}_{a b}}=x_{a} \mathcal{N}_{\partial_{b}}-x_{b} \mathcal{N}_{\partial_{b}}+\sum_{B=1}^{Q}\left(d x_{a} \wedge i_{\partial_{b}} \zeta_{B} \wedge \frac{\partial \Lambda^{\mathbf{m}}}{\partial\left(d \zeta_{B}\right)}-d x_{b} \wedge i_{\partial_{a}} \zeta_{B} \wedge \frac{\partial \Lambda^{\mathbf{m}}}{\partial\left(d \zeta_{B}\right)}\right)
$$

and from (53)

$$
\begin{aligned}
T^{\mathcal{N}}\left(R_{a b}\right) & =T^{\mathcal{N} c}{ }_{a} x_{b}-T^{\mathcal{N} c}{ }_{b} x_{a} \\
& +\star^{-1} \sum_{B=1}^{Q}\left(d x^{c} \wedge d x_{a} \wedge i_{\partial_{b}} \zeta_{B} \wedge \frac{\partial \Lambda^{\mathbf{m}}}{\partial\left(d \zeta_{B}\right)}-d x^{c} \wedge d x_{b} \wedge i_{\partial_{a}} \zeta_{B} \wedge \frac{\partial \Lambda^{\mathbf{m}}}{\partial\left(d \zeta_{B}\right)}\right)
\end{aligned}
$$

Thus the Noether current $\mathcal{N}_{\mathcal{R}_{a b}}$ does not coincide with the moment of Noether linear momentum: $x_{a} \mathcal{N}_{\partial_{b}}-x_{b} \mathcal{N}_{\partial_{b}}$. The additional terms result from the fact that $\mathcal{N}_{v}$ is not $f$ linear in $v$. However although both $T^{\mathcal{B}}$ and $T^{\mathcal{N}}$ give rise to conserved quantities, the lack of $f$-linearity in $T^{\mathcal{N}}$ precludes its use for the definition of stress over curved 2-surfaces.

Equations (81) and (82) remain valid in the presence of Lie-symmetric background fields with $\mathcal{L}_{\mathcal{R}_{a b}} Z_{A}=0$ for $A=1, \ldots, N$ and fixed $a, b$,

\subsection{The Einstein-Hilbert stress-energy-momentum tensor and its associated currents}

In general relativity the variational derivative $\frac{\Delta \Lambda^{\mathrm{m}}}{\Delta g}$ is used to define the algebraically symmetric Einstein-Hilbert stress-energy-momentum tensor $T^{\mathcal{H}} \in \Gamma \otimes^{[\mathbb{F}, \mathbb{F}]} M$ for the theory:

$$
T^{\mathcal{H}}(u, v)=2 \star^{-1}\left(\frac{\Delta \Lambda^{\mathbf{m}}}{\Delta g}:(\widetilde{u} \otimes \widetilde{v})\right) \quad \text { for } \quad u, v \in \Gamma T M
$$

which is manifestly $f$-linear in $u$ and $v$.

Using an arbitrary vector field $v$ on $M$ with a metric $g$, it is convenient to use $T^{\mathcal{H}}$ to define the associated $(n-1)$-form current $\tau_{v}^{\mathcal{H}} \in \Gamma \Lambda^{n-1} M$ by

$$
\tau_{v}^{\mathcal{H}}=\star\left(T^{\mathcal{H}}(v,-)\right)
$$

which is manifestly $f$-linear in $v$. Thus

$$
T^{\mathcal{H}}=\left(\star^{-1} \tau_{X_{a}}^{\mathcal{H}}\right) \otimes e^{a}
$$

where $\left\{e^{a}\right\}$ and $\left\{X_{a}\right\}$ constitute mutually dual frames. From (83) it is also clear that since $g$ is a symmetric tensor, $T^{\mathcal{H}}$ satisfy the algebraic symmetry

$$
T^{\mathcal{H}}(u, v)=T^{\mathcal{H}}(v, u) \quad \text { for all } \quad u, v \in \Gamma T M
$$

(c.f. (59) $)$ and hence $\left\{\tau_{X_{a}}^{\mathcal{H}}\right\}$ satisfy the algebraic symmetry condition

$$
e_{a} \wedge \tau_{X_{b}}^{\mathcal{H}}-e_{b} \wedge \tau_{X_{a}}^{\mathcal{H}}=0
$$

where $e_{a}=g\left(X_{a}, X_{b}\right) e^{b}$. 
In lemma 16 appendix $\mathrm{A}$ it is shown that

$$
\tau_{v}^{\mathcal{H}}=\mathcal{A}_{v}\left(\frac{\Delta \Lambda^{\mathbf{m}}}{\Delta g}, g\right) \quad \text { for all } \quad v \in \Gamma T M
$$

Thus from (43) it follows that in terms of the map $\mathcal{A}_{v}$,

$$
\tau_{v}^{\mathcal{H}}=i_{v} \Lambda^{\mathbf{m}}-\sum_{A=1}^{N} \mathcal{A}_{v}\left(\frac{\Delta \Lambda^{\mathbf{m}}}{\Delta Z_{A}}, Z_{A}\right)-\sum_{B=1}^{Q}\left(i_{v} \zeta_{B} \wedge \frac{\partial \Lambda^{\mathbf{m}}}{\partial \zeta_{B}}+i_{v} d \zeta_{B} \wedge \frac{\partial \Lambda^{\mathbf{m}}}{\partial\left(d \zeta_{B}\right)}\right)
$$

Let the exterior covariant derivative of $\tau_{v}^{\mathcal{H}}$ be defined by

$$
\left(D \tau^{\mathcal{H}}\right)_{v}=\left(i_{v} e^{a}\right) d \tau_{X_{a}}^{\mathcal{H}}-i_{v} d e^{a} \wedge \tau_{X_{a}}^{\mathcal{H}}
$$

This corresponds to the standard Levi-Civita covariant derivative in the case of a torsion free metric-compatible connection $\nabla$ and $\left(D \tau^{\mathcal{H}}\right)_{v}=0$ implies $\nabla \cdot T^{\mathcal{H}}=0$.

In lemma 17 appendix $\mathrm{A}$ it is shown that in terms of the map $\mathcal{B}_{v}$,

$$
\left(D \tau^{\mathcal{H}}\right)_{v}=-\mathcal{B}_{v}\left(\frac{\Delta \Lambda^{\mathrm{m}}}{\Delta g}, g\right)
$$

Hence for a system on $\Lambda^{\mathbf{m}}$-shell one has, from (46),

$$
\left(D \tau^{\mathcal{H}}\right)_{v}=\sum_{A=1}^{N} \mathcal{B}_{v}\left(\frac{\Delta \Lambda^{\mathbf{m}}}{\Delta Z_{A}}, Z_{A}\right)
$$

It is worth stressing that, in general, even if $N=0$ this relation is not a conservation law since in general $\left(D \tau^{\mathcal{H}}\right)_{v} \neq d \tau_{v}^{\mathcal{H}}$. However, if $K \in \Gamma T M$ is a Killing vector field, $\mathcal{L}_{K} g=0$, then from (88), (170) and (91)

$$
d \tau_{K}^{\mathcal{H}}=d\left(\mathcal{A}_{K}\left(\frac{\Delta \Lambda^{\mathrm{m}}}{\Delta g}, g\right)\right)=-\mathcal{B}_{K}\left(\frac{\Delta \Lambda^{\mathbf{m}}}{\Delta g}, g\right)=\left(D \tau^{\mathcal{H}}\right)_{K}
$$

i.e.

$$
\left(D \tau^{\mathcal{H}}\right)_{K}=d \tau_{K}^{\mathcal{H}}
$$

From the definitions above, the relationship between the Einstein-Hilbert, Noether and Belinfante-Rosenfeld currents is given by

$$
\tau_{v}^{\mathcal{H}}+\sum_{A=1}^{N} \mathcal{A}_{v}\left(\frac{\Delta \Lambda^{\mathbf{m}}}{\Delta Z_{A}}, Z_{A}\right)=\tau_{v}^{\mathcal{B}}=\mathcal{N}_{v}+d S_{v}
$$

As stated in (69) $\tau_{K}^{\mathcal{B}}$ and $\mathcal{N}_{K}$ give rise to conserved currents associated with each Killing vector field $K$ in the presence of the Lie-symmetric background fields $\{\boldsymbol{Z}\}$. By contrast, from (93) and (91), in the presence of arbitrary background fields, whether Lie-symmetric or not,

$$
d \tau_{K}^{\mathcal{H}}=\sum_{A=1}^{N} \mathcal{B}_{K}\left(\frac{\Delta \Lambda^{\mathbf{m}}}{\Delta Z_{A}}, Z_{A}\right)
$$


which in general does not vanish. However in general, as stated above, unlike $\tau_{K}^{\mathcal{H}}$ neither $\tau_{K}^{\mathcal{B}}$ nor $\mathcal{N}_{K}$ possesses the algebraic symmetry condition (159), (87) in the presence of any background fields.

If there are no background tensor fields $\{\boldsymbol{Z}\}$, i.e. $N=0$ then from (94)

$$
\tau_{v}^{\mathcal{B}}=\tau_{v}^{\mathcal{H}}=\mathcal{N}_{v}+d S_{v} \quad \text { for all } \quad v \in \Gamma T M
$$

and hence if $K$ is Killing from (69)

$$
d \tau_{K}^{\mathcal{H}}=d \tau_{K}^{\mathcal{B}}=d \mathcal{N}_{K}=0
$$

In this case, since $\tau_{v}^{\mathcal{H}}$ satisfies the algebraically symmetry condition it follows from (96) that $\tau_{v}^{\mathcal{B}}$ also does. However in general $\mathcal{N}_{v}$ does not.

In the context of model (12) the Einstein-Hilbert stress-energy-momentum tensor $T^{\mathcal{H}}$ is given by (83) by evaluating the derivative $\frac{\Delta \Lambda^{\mathbf{m}}}{\Delta g}$ at

$$
\left(g, Z^{\mathrm{de}}, Z^{\mathrm{db}}, Z^{\mathrm{he}}, Z^{\mathrm{hb}}, V, d A\right)=\left(g_{0}, Y^{\mathrm{de}}, Y^{\mathrm{db}}, Y^{\mathrm{he}}, Y^{\mathrm{hb}}, W, d A\right)
$$

where $g_{0}$ is an arbitrary background metric with associated Hodge map $\star_{0}$ and the 4velocity of the medium $W$ satisfies $g_{0}(W, W)=-1$. The $Y^{\mathrm{I}} \in\left\{Y^{\mathrm{de}}, Y^{\mathrm{db}}, Y^{\mathrm{he}}, Y^{\mathrm{hb}}\right\}$ are spatial with respect to $W$, i.e. $Y^{\mathrm{I}}\left(g_{0}(W,-)\right)=0$ and $i_{W} Y^{\mathrm{I}}(\alpha)=0$ and satisfy the adjoint properties with respect to $g_{0}$ :

$$
\begin{gathered}
\alpha \wedge \star_{0} Y^{\mathrm{de}}(\beta)=\beta \wedge \star_{0} Y^{\mathrm{de}}(\alpha), \quad \alpha \wedge \star_{0} Y^{\mathrm{hb}}(\beta)=\beta \wedge \star_{0} Y^{\mathrm{hb}}(\alpha) \\
\text { and } \alpha \wedge \star_{0} Y^{\mathrm{db}}(\beta)=-\beta \wedge \star_{0} Y^{\mathrm{he}}(\alpha)
\end{gathered}
$$

for all $\alpha, \beta \in \Gamma \Lambda^{1} M$. The tensor $T^{\mathcal{H}}$ is the Abraham stress-energy-momentum tensor for electromagnetic fields in (moving) media [2, 3].

\section{Caveats and Conclusions}

The results in this article have direct relevance to the construction of variational formulations of field systems in spacetime where complex microscopic interactions are represented by phenomenological macroscopic constitutive relations between the dynamic fields $\{\boldsymbol{\zeta}\}$. It is tacitly assumed that the variational field equations based on the matter action $\int_{M} \Lambda^{\mathrm{m}}$ admit such $\{\boldsymbol{\zeta}\}$ as non-trivial physically acceptable solutions. This condition may impose constraints on the constitutive modelling. For example if

$$
\Lambda^{\mathbf{m}}(g, J, A)=\frac{1}{2} d A \wedge \star d A+A \wedge J
$$

where $A \in \Gamma \Lambda^{1} \mathcal{M}$ is dynamical and $J \in \Gamma \Lambda^{3} \mathcal{M}$ is a background field then the $\Lambda^{\mathrm{m}}$ matter shell condition $\frac{\delta \Lambda^{\mathrm{m}}}{\delta A}=0$ implies $d J=0$. The physical interpretation of such models for continuous media can then be facilitated by finding conserved currents associated with continuous symmetries.

For the class of theories described by a diffeomorphism invariant matter action $\int_{M} \Lambda^{\mathbf{m}}$, where $\Lambda^{\mathbf{m}}$ in general depends on a background gravitation field, a collection of background 
tensors $\{\boldsymbol{Z}\}$ and matter fields $\{\boldsymbol{\zeta}\}$ the differential forms $\tau_{v}^{\mathcal{H}}, \tau^{\mathcal{B}}$ and $\mathcal{N}_{v}$ are related by

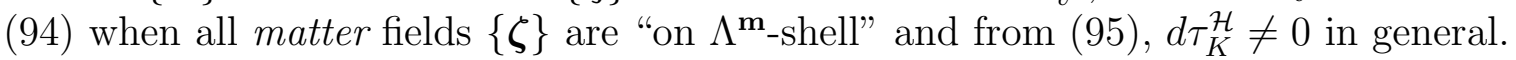

However, in Einstein's theory

$$
\Lambda^{\mathbf{T}}=\mathcal{R} \star 1+\Lambda^{\mathbf{m}}\left(g, Z_{1}, \ldots, Z_{N}, \zeta_{1}, d \zeta_{1}, \ldots, \zeta_{Q}, d \zeta_{Q}\right)
$$

where $\mathcal{R}$ is the curvature scalar derived from $g$. If the metric $g$ is dynamical as well as the matter fields $\{\boldsymbol{\zeta}\}$ one has the additional on-shell Einstein equation

$$
\mathcal{G}_{v}=\tau_{v}^{\mathcal{H}}
$$

where the Einstein $(n-1)$-form $\mathcal{G}_{v} \in \Gamma \Lambda^{n-1} M$ for $v \in \Gamma T M$ is [1]

$$
\mathcal{G}_{v}=\frac{1}{2} R\left(X_{c}, X_{d}, X_{b}, e^{a}\right) e^{c} \wedge e^{d} \wedge i_{v} \star\left(e_{a} \wedge e^{b}\right)
$$

Since the Bianchi identity for $\mathcal{G}_{v}$ yields $(D \mathcal{G})_{v}=0$ for all $v \in \Gamma T M$, (101) implies

$$
\left(D \tau^{\mathcal{H}}\right)_{v}=0
$$

Then from (93), if $K$ is Killing one has the conservation law

$$
d \tau_{K}^{\mathcal{H}}=0
$$

and hence from (92) and (103)

$$
\sum_{A=1}^{N} \mathcal{B}_{v}\left(\frac{\Delta \Lambda^{\mathbf{m}}}{\Delta Z_{A}}, Z_{A}\right)=0
$$

This relation may impose constraints on the dependence of $\Lambda^{\mathbf{m}}$ on the non-gravitational background structure $\{\boldsymbol{Z}\}$. For example in the analysis of a gravitational wave propagating in a material medium.

In physical applications it is often convenient to break a system into weakly interacting subsystems in order to analyse the dynamics of subsystems perturbatively. However care is required in extending the consequences of diffeomorphism invariance for $\Lambda^{\mathrm{m}}$ to topforms $\Lambda^{s}$ describing diffeomorphic invariant sub-systems. For each $\Lambda^{s}$ one may define an associated Noether form $\mathcal{N}_{v}^{s}$ :

$$
\mathcal{N}_{v}^{s}=i_{v} \Lambda^{s}-\sum_{B=1}^{Q} \mathcal{L}_{v} \zeta_{B} \wedge \frac{\partial \Lambda^{s}}{\partial\left(d \zeta_{B}\right)}
$$

Thus

$$
\mathcal{N}_{v}=\sum_{s} \mathcal{N}_{v}^{s}
$$

Similarly

$$
\tau_{v}^{\mathcal{H}}=\sum_{s} \tau_{v}^{s, \mathcal{H}}
$$


where

$$
T^{s, \mathcal{H}}(u, v)=2 \star^{-1}\left(\frac{\Delta \Lambda^{s}}{\Delta g}:(\widetilde{u} \otimes \widetilde{v})\right) \quad \text { for } \quad u, v \in \Gamma T M
$$

and

$$
\tau_{v}^{s, \mathcal{H}}=\star\left(T^{s, \mathcal{H}}(v,-)\right)
$$

When all matter fields $\{\boldsymbol{\zeta}\}$ are "on $\Lambda^{\mathrm{m}}$-shell" they will not necessarily be "on $\Lambda^{s}$-shell" and one cannot associate conserved currents with subsystems, in general. This is simply a reflection of the interaction between subsystems in situations where $g$ and $\{\boldsymbol{Z}\}$ are background fields. However, given some decomposition $\Lambda^{\mathrm{m}}=\sum_{s} \Lambda^{s}$, suppose that for some $s=s_{0}$ there exists a subset of matter fields $\left\{\zeta_{B}\right\}$ such that $\frac{\delta \Lambda^{s_{0}}}{\delta \zeta_{B}}=0$ for $B=$ $1 \ldots Q_{0}$. In this situation one may regard the quantities $\zeta_{B}, d \zeta_{B}$ for $B=Q_{0}+1 \ldots Q$ as background fields to supplement those in $\boldsymbol{Z}$. The analysis in this paper is then applicable by disregarding all $\Lambda^{s}$ with $s \neq s_{0}$, replacing $\Lambda^{\mathrm{m}}$ by $\Lambda^{s_{0}}$ and disregarding the equations $\frac{\delta \Lambda^{s_{0}}}{\delta \zeta_{B}} \neq 0$ for $B=Q_{0}+1 \ldots Q$.

Maps such as $T^{\mathcal{N}}, T^{\mathcal{B}}$ and $T^{\mathcal{H}}$ have been traditionally used to construct densities of field energy and linear momentum in Minkowski spacetime. Applied to closed systems in the presence of the Lie-symmetric background fields $\{g, \boldsymbol{Z}\}$ this procedure is strictly only meaningful for $T^{\mathcal{N}}$ and $T^{\mathcal{B}}$ since they alone give rise to conserved currents in Minkowski spacetime. Furthermore only $T^{\mathcal{B}}$ can meaningfully be used to describe stress and angular momentum in Minkowski spacetime. In a general spacetime with a non-dynamic metric neither $T^{\mathcal{B}}$, $T^{\mathcal{H}}$ nor $T^{\mathcal{N}}$ give rise to conserved Killing currents unless $\mathcal{L}_{K} g=0$ and $\mathcal{L}_{K} Z_{A}=0$ for all $A$.

In any background metric, the model described by (5) yields a particular $T^{\mathcal{B}}$ known as the non-symmetric Minkowski stress-energy-momentum tensor and $T^{\mathcal{H}}$ as its symmetrised version.

The model (12) yields a symmetric $T^{\mathcal{H}}$ known as the Abraham stress-energy-momentum tensor (which for a general Lie-symmetric $\boldsymbol{Z}$ does not generate conserved Killing currents). This model does however yield $T^{\mathcal{B}}$ that coincides with the non-symmetric Minkowski stress-energy-momentum tensor. This sheds light on the relationship between the Abraham and non-symmetric Minkowski stress-energy-momentum tensors. The precise relation between these two tensors, in this model follows from (94) as

$$
\begin{aligned}
\tau_{v}^{\mathcal{B}}=\tau_{v}^{\mathcal{H}}+ & \mathcal{A}_{v}\left(\frac{\Delta \Lambda^{\mathrm{m}}}{\Delta V}, V\right)+\mathcal{A}_{v}\left(\frac{\Delta \Lambda^{\mathrm{m}}}{\Delta Z^{\mathrm{de}}}, Z^{\mathrm{de}}\right) \\
& +\mathcal{A}_{v}\left(\frac{\Delta \Lambda^{\mathrm{m}}}{\Delta Z^{\mathrm{db}}}, Z^{\mathrm{db}}\right)+\mathcal{A}_{v}\left(\frac{\Delta \Lambda^{\mathrm{m}}}{\Delta Z^{\mathrm{he}}}, Z^{\mathrm{he}}\right)+\mathcal{A}_{v}\left(\frac{\Delta \Lambda^{\mathrm{m}}}{\Delta Z^{\mathrm{hb}}}, Z^{\mathrm{hb}}\right)
\end{aligned}
$$

where all tensors are evaluated at the point

$$
\left(g, Z^{\mathrm{de}}, Z^{\mathrm{db}}, Z^{\mathrm{he}}, Z^{\mathrm{hb}}, V, d A\right)=\left(g_{0}, Y^{\mathrm{de}}, Y^{\mathrm{db}}, Y^{\mathrm{he}}, Y^{\mathrm{hb}}, W, d A\right)
$$

Conclusions drawn from different models are directly related to the epistemology used to describe the linear and angular momentum of light in unbounded media described by background constitutive tensor fields in background gravitational fields. 
Thus the physical consequences of any model based on a diffeomorphism invariant action used to describe the dynamics of matter fields in the presence of a specified selfconsistent non-dynamic background depend not only on the action for the model but also on a choice of objects, such as $T^{\mathcal{H}}, T^{\mathcal{B}}$ or $T^{\mathcal{N}}$, that are adopted to define conserved quantities, including field energy, momentum and angular momentum. Furthermore these may only acquire physical cogency in the presence of sufficient Lie symmetry.

\section{Acknowledgments}

J.G. and R.W.T. are grateful to STFC and the Cockcroft Inistitute for support (STFC ST/G008248/1). Y.N.O. acknowledges partial support by the German-Israeli Foundation for Scientific Research and Development (GIF), Research Grant No. 1078-107.14/2009. This work is the outcome of discussions initiated by the author's attendence at the 475th Wilhelm \& Else Heraeus Seminar on "Problems and Developments of Classical Electrodynamics" Bad Honnef March 2011, organised by Domenico Giulini and Volker Perlick.

\section{References}

[1] I.M. Benn and R.W. Tucker. An introduction to spinors and geometry with applications in physics. Hilger, 1987. Eqns: (6.4.11) and (6.8.3).

[2] T. Dereli, J. Gratus, and R.W. Tucker. The covariant description of electromagnetically polarizable media. Physics Letters A, 361(3):190-193, 2007.

[3] T. Dereli, J. Gratus, and R.W. Tucker. New perspectives on the relevance of gravitation for the covariant description of electromagnetically polarizable media. Journal of Physics A: Mathematical and Theoretical, 40:5695, 2007.

[4] H. Goldstein. Classical Mechanics. Addison-Wesley, 1959.

[5] M.J. Gotay and J.E. Marsden. Stress-energy-momentum tensors and the BelinfanteRosenfeld formula. Contemp. Math, 132:367-391, 1992.

[6] F.W. Hehl. Maxwell's equations in Minkowski's world: their premetric generalization and the electromagnetic energy-momentum tensor. Annalen der Physik, 17(910):691-704, 2008.

[7] F.W. Hehl, J.D. McCrea, E.W. Mielke, and Y. Ne'eman. Metric-affine gauge theory of gravity: field equations, noether identities, world spinors, and breaking of dilation invariance. Physics Reports, 258(1-2):1-171, 1995.

[8] F.W. Hehl and Y.N. Obukhov. Foundations of classical electrodynamics: Charge, flux, and metric. Birkhauser, Boston, 2003.

[9] H. Minkowski. Die Grundgleichungen für die elektromagnetischen Vorgänge in bewegten Körpern. Mathematische Annalen, 68(4):472-525, 1910. Also reprinted in: Zwei Abhandlungen über die Grundgleichungen der Elektrodynamik (B.G. Teubner Verlag, Leipzig und Berlin, 1910). 
[10] Y.N. Obukhov. Electromagnetic energy and momentum in moving media. Annalen der Physik, 17(9-10):830-851, 2008.

[11] D.E. Soper. Classical field theory. John Wiley and Sons, Inc., New York, 1976.

[12] R.W. Tucker and T.J. Walton. An intrinsic approach to forces in magnetoelectric media. Nuovo Cimento Della Societa Italiana Di Fisica C-Geophysics And Space Physics, 32:205-229, 2009.

[13] R.M. Wald. General relativity. University of Chicago Press, 1984.

\section{A Mathematical Details of results used in the text}

Lemma 4. On an $n$ dimensional manifold $M$, given the (co-vector valued) forms $\alpha_{w} \in$ $\Gamma \Lambda^{n-1} M$ and $\beta_{w} \in \Gamma \Lambda^{n} M$ which are 'f'-linear in $w$ such that

$$
d\left(\alpha_{w}\right)=\beta_{w}
$$

for all $w \in \Gamma T M$ with compact support then $\alpha_{w}=0$ and $\beta_{w}=0$.

Proof. Let $w=w^{a} X_{a}, \alpha_{a}=\alpha_{X_{a}}$ and $\beta_{a}=\beta_{X_{a}}$. Given any subset $U \subset M$ with boundary $\partial U$

$$
\int_{\partial U} \iota^{\star}\left(w^{a} \alpha_{a}\right)=\int_{U} w^{a} \beta_{a}
$$

where $\iota: \partial U \rightarrow M$ is the embedding. Assume first that $w$ has support away from the boundary $\partial U$ and in an arbitrary small region then

$$
\int_{U} w^{a} \beta_{a}=0
$$

and hence $\beta_{a}=0$. Thus

$$
\int_{\partial U} w^{a} \iota^{\star}\left(\alpha_{a}\right)=0
$$

For all subsets $U \subset M$. Considering $w$ to have a support on a small set about a point in the boundary implies $\iota^{\star} \alpha_{a}=0$. Since we can choose any $\partial U$ we show that all the components of $\alpha_{a}=0$ and hence $\alpha_{a}=0$.

Lemma 5. (40) implies (41) and (42).

Proof. Since (40) is true for all $v \in \Gamma T M$ it is true for all $v=w \in \Gamma T M$ with compact support. Thus (41) and (42) follow from lemma 4 setting

$$
\alpha_{w}=i_{w} \Lambda^{\mathbf{m}}-\sum_{C=0}^{N+2 Q} \mathcal{A}_{w}\left(\frac{\Delta \Lambda^{\mathrm{m}}}{\Delta \mathcal{Z}_{C}}, \mathcal{Z}_{C}\right) \quad \text { and } \quad \beta_{w}=-\sum_{C=0}^{N+2 Q} \mathcal{B}_{w}\left(\frac{\Delta \Lambda^{\mathbf{m}}}{\Delta \mathcal{Z}_{C}}, \mathcal{Z}_{C}\right)
$$

Lemma 6. For any $\alpha \in \Gamma \Lambda^{p} M$ we have (24) 
Proof. Setting

$$
\alpha=\sum_{I_{1}<\ldots<I_{p}} \alpha_{I_{1} \cdots I_{p}} e^{I_{1}} \wedge \cdots \wedge e^{I_{p}}
$$

From (2) and (22) we have

$$
\begin{aligned}
\alpha: \mathcal{V}= & \left(\frac{1}{p !} \sum_{I_{1}<\ldots<I_{p}} \sum_{\sigma \in S_{p}} \alpha_{I_{1} \cdots I_{p}} \epsilon(\sigma) e^{\sigma\left(I_{1}\right)} \otimes \cdots \otimes e^{\sigma\left(I_{p}\right)}\right) \\
& :\left(\frac{1}{p !} \sum_{J_{1}<\ldots<J_{p}} \mathcal{V}^{J_{1} \cdots J_{p}} \sum_{\rho \in S_{p}} \epsilon(\rho) X_{\rho\left(J_{1}\right)} \otimes \cdots \otimes X_{\rho\left(J_{p}\right)}\right) \\
= & \frac{1}{(p !)^{2}} \sum_{I_{1}<\ldots<I_{p}} \sum_{J_{1}<\ldots<J_{p}} \sum_{\sigma \in S_{p}} \sum_{\rho \in S_{p}} \alpha_{I_{1} \cdots I_{p}} \mathcal{V}^{J_{1} \cdots J_{p}} \epsilon(\sigma) \epsilon(\rho) \delta_{\rho\left(J_{1}\right)}^{\sigma\left(I_{1}\right)} \cdots \delta_{\rho\left(J_{p}\right)}^{\sigma\left(I_{p}\right)} \\
= & \frac{1}{p !} \sum_{I_{1}<\ldots<I_{p}} \sum_{J_{1}<\ldots<J_{p}} \alpha_{I_{1} \cdots I_{p}} \mathcal{V}^{J_{1} \cdots J_{p}} \delta_{J_{1}}^{I_{1}} \cdots \delta_{J_{p}}^{I_{p}} \\
= & \frac{1}{p !} \sum_{I_{1}<\ldots<I_{p}} \alpha_{I_{1} \cdots I_{p}} \mathcal{V}^{I_{1} \cdots I_{p}}
\end{aligned}
$$

while

$$
\begin{aligned}
i_{\mathcal{V}} \alpha & =\frac{1}{p !} \sum_{J_{1}<\ldots<J_{p}} \mathcal{V}^{J_{1} \cdots J_{p}} i_{X_{J_{p}}} \cdots i_{X_{J_{1}}}\left(\sum_{I_{1}<\ldots<I_{p}} \alpha_{I_{1} \cdots I_{p}} e^{I_{1}} \wedge \cdots \wedge e^{I_{p}}\right) \\
& =\frac{1}{p !} \sum_{J_{1}<\ldots<J_{p}} \sum_{I_{1}<\ldots<I_{p}} \alpha_{I_{1} \cdots I_{p}} \mathcal{V}^{J_{1} \cdots J_{p}} i_{X_{J_{p}}} \cdots i_{X_{J_{1}}} e^{I_{1}} \wedge \cdots \wedge e^{I_{p}} \\
& =\frac{1}{p !} \sum_{J_{1}<\ldots<J_{p}} \sum_{I_{1}<\ldots<I_{p}} \alpha_{I_{1} \cdots I_{p}} \mathcal{V}^{J_{1} \cdots J_{p}} \delta_{J_{1}}^{I_{1}} \cdots \delta_{J_{p}}^{I_{p}} \\
& =\frac{1}{p !} \sum_{I_{1}<\ldots<I_{p}} \alpha_{I_{1} \cdots I_{p}} \mathcal{V}^{I_{1} \cdots I_{p}}
\end{aligned}
$$

Lemma 7. For any $\alpha \in \Gamma \Lambda^{p} M$, one has (21)

Proof. For $0 \leq q \leq p$ we have since $\left(i_{v_{1}} \cdots i_{v_{q}} \alpha\right) \wedge\left(i_{v_{q+1}} \cdots i_{v_{p}} \Omega\right) \in \Gamma \Lambda^{n} M$

$$
\begin{aligned}
\left(i_{v_{1}} \cdots i_{v_{q}} \alpha\right) & \wedge\left(i_{v_{q+1}} \cdots i_{v_{p}} \Omega\right) \\
= & (-1)^{q-1} i_{v_{q}}\left(i_{v_{1}} \cdots i_{v_{q-1}} \alpha\right) \wedge\left(i_{v_{q+1}} \cdots i_{v_{p}} \Omega\right) \\
= & (-1)^{q-1}(-1)^{(p-q)}\left(i_{v_{1}} \cdots i_{v_{q-1}} \alpha\right) \wedge i_{v_{q}}\left(i_{v_{q+1}} \cdots i_{v_{p}} \Omega\right) \\
= & (-1)^{p-1}\left(i_{v_{1}} \cdots i_{v_{q-1}} \alpha\right) \wedge\left(i_{v_{q}} \cdots i_{v_{p}} \Omega\right)
\end{aligned}
$$


Hence from (18) and (24)

$$
\begin{aligned}
(\Omega \otimes \mathcal{V}): \alpha & =(\mathcal{V}: \alpha) \Omega=\left(i_{\mathcal{V}} \alpha\right) \Omega=\frac{1}{p !} \sum_{I_{1}<\cdots<I_{p}} \mathcal{V}^{I_{1} \cdots I_{p}}\left(i_{X_{I_{p}}} \cdots i_{X_{I_{1}}} \alpha\right) \wedge \Omega \\
& =\frac{1}{p !} \sum_{I_{1}<\cdots<I_{p}} \mathcal{V}^{I_{1} \cdots I_{p}}(-1)^{p(p-1)} \alpha \wedge\left(i_{X_{I_{p}}} \cdots i_{X_{I_{1}}} \Omega\right) \\
& =\alpha \wedge\left(\frac{1}{p !} \sum_{I_{1}<\cdots<I_{p}} \mathcal{V}^{I_{1} \cdots I_{p}} i_{X_{I_{p}}} \cdots i_{X_{I_{1}}} \Omega\right) \\
& =\alpha \wedge i_{\mathcal{V}} \Omega
\end{aligned}
$$

hence (21).

Lemma 8. (27) holds.

Proof. From (20) and (25)

$$
\begin{aligned}
\int_{M} & \beta \\
= & \left.\wedge \frac{\delta \Lambda^{\mathbf{m}}}{\delta \zeta_{B}}\right|_{\varepsilon=0} \int_{M} \Lambda^{\mathbf{m}}\left(g, Z_{1}, \ldots, Z_{N}, \zeta_{1}, d \zeta_{1}, \ldots, \zeta_{B}+\varepsilon \beta, d \zeta_{B}+\varepsilon d \beta, \ldots, \zeta_{Q}, d \zeta_{Q}\right) \\
& =\int_{M}\left(\beta \wedge \frac{\partial \Lambda^{\mathbf{m}}}{\partial \zeta_{B}}+d \beta \wedge \frac{\partial \Lambda^{\mathbf{m}}}{\partial\left(d \zeta_{B}\right)}\right) \\
& =\int_{M}\left(\beta \wedge \frac{\partial \Lambda^{\mathbf{m}}}{\partial \zeta_{B}}+d\left(\beta \wedge \frac{\partial \Lambda^{\mathbf{m}}}{\partial \zeta_{B}}\right)-(-1)^{p_{B}} \beta \wedge d\left(\frac{\partial \Lambda^{\mathbf{m}}}{\partial \zeta_{B}}\right)\right) \\
& =\int_{M} \beta \wedge\left(\frac{\partial \Lambda^{\mathbf{m}}}{\partial \zeta_{B}}+(-1)^{p_{B}+1} d\left(\frac{\partial \Lambda^{\mathbf{m}}}{\partial\left(d \zeta_{B}\right)}\right)\right)
\end{aligned}
$$

since $\beta$ has compact support. Since this is true for all $\beta$ then (27) follows.

Lemma 9. For any two forms $\alpha \in \Gamma \Lambda^{n-p} M$ and $\beta \in \Gamma \Lambda^{p} M$ then

$$
\mathcal{L}_{v} \beta \wedge \alpha=d\left(i_{v} \beta \wedge \alpha\right)+(-1)^{p} i_{v} \beta \wedge d \alpha+i_{v} d \beta \wedge \alpha
$$

Proof.

$$
\mathcal{L}_{v} \beta \wedge \alpha=d i_{v} \beta \wedge \alpha+i_{v} d \beta \wedge \alpha=d\left(i_{v} \beta \wedge \alpha\right)+(-1)^{p} i_{v} \beta \wedge d \alpha+i_{v} d \beta \wedge \alpha
$$

Lemma 10. For any tensors $Z \in \Gamma \otimes^{s} M$ and $\Psi \in \Gamma\left(\Lambda^{n} M \otimes \otimes^{\bar{s}} M\right)$ then

$$
\Psi:\left(\mathcal{L}_{v} Z\right)=d\left(\mathcal{A}_{v}(\Psi, Z)\right)+\mathcal{B}_{v}(\Psi, Z)
$$

Furthermore this decomposition is unique in the sense that if

$$
\Psi:\left(\mathcal{L}_{v} Z\right)=d \alpha_{v}+\beta_{v}
$$

where $\alpha_{v}$ and $\beta_{v}$ are 'f'-linear in $v$ then

$$
\alpha_{v}=\mathcal{A}_{v}(\Psi, Z) \quad \text { and } \quad \beta_{v}=\mathcal{B}_{v}(\Psi, Z)
$$


Proof. First using (36) we prove that (113) for $f \in \Gamma \otimes^{()} M$

$$
\Omega:\left(\mathcal{L}_{v} f\right)=\Omega v(f)=d\left(\mathcal{A}_{v}(\Omega, f)\right)+\mathcal{B}_{v}(\Omega, f)
$$

Using (37) this is true for 1 -forms $\alpha \in \Gamma \otimes^{[\mathbb{F}]} M$

$$
\begin{aligned}
(\Omega \otimes u):\left(\mathcal{L}_{v} \alpha\right) & =\Omega\left(i_{u} \mathcal{L}_{v} \alpha\right)=(-1)^{n+1} i_{u} \Omega \wedge d i_{v} \alpha+(-1)^{n+1} i_{u} \Omega \wedge i_{v} d \alpha \\
& =d\left(i_{u} \Omega \wedge i_{v} \alpha\right)-d i_{u} \Omega \wedge i_{v} \alpha+(-1)^{n+1} i_{u} \Omega \wedge i_{v} d \alpha \\
& =d\left(\mathcal{A}_{v}(\Omega \otimes u, \alpha)\right)+\mathcal{B}_{v}(\Omega \otimes u, \alpha)
\end{aligned}
$$

Now using (38) we show that for vectors $u \in \Gamma \otimes^{[\mathbb{V}]} M$

$$
\begin{aligned}
(\Omega \otimes \alpha) & :\left(\mathcal{L}_{v} u\right)=\Omega\left(\alpha\left(\mathcal{L}_{v} u\right)\right)=\Omega(v(\alpha(u)))-\Omega\left(i_{u} \mathcal{L}_{v} \alpha\right) \\
& =\Omega(v(\alpha(u)))-d\left(i_{u} \Omega \wedge i_{v} \alpha\right)+d i_{u} \Omega \wedge i_{v} \alpha-(-1)^{n+1} i_{u} \Omega \wedge i_{v} d \alpha \\
& =d\left(\mathcal{A}_{v}(\Omega \otimes \alpha, u)\right)+\mathcal{B}_{v}(\Omega \otimes \alpha, u)
\end{aligned}
$$

Using (39) it follows that (113) is true across tensor products. Assuming it true for $Z_{1}, Z_{2}$ then

$$
\begin{aligned}
(\Omega \otimes & \left.\Phi_{1} \otimes \Phi_{2}\right):\left(\mathcal{L}_{v}\left(Z_{1} \otimes Z_{2}\right)\right) \\
= & \left(\Omega \otimes \Phi_{1} \otimes \Phi_{2}\right):\left(\mathcal{L}_{v} Z_{1} \otimes Z_{2}\right)+\left(\Omega \otimes \Phi_{1} \otimes \Phi_{2}\right):\left(Z_{1} \otimes \mathcal{L}_{v} Z_{2}\right) \\
= & \Omega\left(\Phi_{1}: \mathcal{L}_{v} Z_{1}\right)\left(\Phi_{2}: Z_{2}\right)+\Omega\left(\Phi_{1}: Z_{1}\right)\left(\Phi_{2}: \mathcal{L}_{v} Z_{2}\right) \\
= & \left(\Phi_{2}: Z_{2}\right)\left(\Omega \otimes \Phi_{1}\right):\left(\mathcal{L}_{v} Z_{1}\right)+\left(\Phi_{1}: Z_{1}\right)\left(\Omega \otimes \Phi_{2}\right):\left(\mathcal{L}_{v} Z_{2}\right) \\
= & d\left(\mathcal{A}_{v}\left(\left(\Phi_{2}: Z_{2}\right) \Omega \otimes \Phi_{1}, Z_{1}\right)\right)+\mathcal{B}_{v}\left(\left(\Phi_{2}: Z_{2}\right) \Omega \otimes \Phi_{1}, Z_{1}\right) \\
& +d\left(\mathcal{A}_{v}\left(\left(\Phi_{1}: Z_{1}\right) \Omega \otimes \Phi_{2}, Z_{2}\right)\right)+\mathcal{B}_{v}\left(\left(\Phi_{1}: Z_{1}\right) \Omega \otimes \Phi_{2}, Z_{2}\right) \\
= & d\left(\mathcal{A}_{v}\left(\Omega \otimes \Phi_{1} \otimes \Phi_{2}, Z_{1} \otimes Z_{2}\right)\right)+\mathcal{B}_{v}\left(\Omega \otimes \Phi_{1} \otimes \Phi_{2}, Z_{1} \otimes Z_{2}\right)
\end{aligned}
$$

Hence (113) is true for all tensors $Z$.

If (114) is true then from (113) one has

$$
d\left(\mathcal{A}_{v}(\Psi, Z)-\alpha_{v}\right)=-\left(\mathcal{B}_{v}(\Psi, Z)-\beta_{v}\right)
$$

and hence from lemma 4 (115) follows.

Lemma 11. Given $\Omega \otimes \mathcal{V} \in \Gamma\left(\Lambda^{n} M \otimes \otimes \otimes^{[\mathbb{V}, \ldots, \mathbb{V}]} M\right)$ where $\mathcal{V}$ is antisymmetric and $[\mathbb{V}, \ldots, \mathbb{V}]$ has length $p$ and $\beta \in \Gamma \Lambda^{p} M$ then

$$
\mathcal{A}_{v}(\beta, \Omega \otimes \mathcal{V})=i_{v} \beta \wedge i_{\mathcal{V}} \Omega
$$

and

$$
\mathcal{B}_{v}(\beta, \Omega \otimes \mathcal{V})=(-1)^{p} i_{v} \beta \wedge d i_{\mathcal{V}} \Omega+i_{v} d \beta \wedge i_{\mathcal{V}} \Omega
$$

Proof. From (113), (21) and (112) we have

$$
\begin{array}{r}
d\left(\mathcal{A}_{v}(\beta, \Omega \otimes \mathcal{V})\right)+\mathcal{B}_{v}(\beta, \Omega \otimes \mathcal{V})=(\Omega \otimes \mathcal{V}) \vdots\left(\mathcal{L}_{v} \beta\right)=\left(\mathcal{L}_{v} \beta\right) \wedge i_{\mathcal{V}} \Omega \\
=d\left(i_{v} \beta \wedge i_{\mathcal{V}} \Omega\right)+(-1)^{p} i_{v} \beta \wedge d i_{\mathcal{V}} \Omega+i_{v} d \beta \wedge i_{\mathcal{V}} \Omega
\end{array}
$$

Now apply lemma 4. 
Lemma 12. If the system is on $\Lambda^{\mathbf{m}}$-shell then (45) and (46) hold.

Proof. From (29)

$$
\begin{aligned}
i_{v} \zeta_{B} \wedge \frac{\partial \Lambda^{\mathbf{m}}}{\partial \zeta_{B}} & +i_{v} d \zeta_{B} \wedge \frac{\partial \Lambda^{\mathbf{m}}}{\partial\left(d \zeta_{B}\right)} \\
& =(-1)^{p_{B}} i_{v} \zeta_{B} \wedge d\left(\frac{\partial \Lambda^{\mathbf{m}}}{\partial\left(d \zeta_{B}\right)}\right)+i_{v} d \zeta_{B} \wedge \frac{\partial \Lambda^{\mathbf{m}}}{\partial\left(d \zeta_{B}\right)} \\
& =-d\left(i_{v} \zeta_{B} \wedge \frac{\partial \Lambda^{\mathbf{m}}}{\partial\left(d \zeta_{B}\right)}\right)+d i_{v} \zeta_{B} \wedge \frac{\partial \Lambda^{\mathbf{m}}}{\partial\left(d \zeta_{B}\right)}+i_{v} d \zeta_{B} \wedge \frac{\partial \Lambda^{\mathbf{m}}}{\partial\left(d \zeta_{B}\right)} \\
& =-d\left(i_{v} \zeta_{B} \wedge \frac{\partial \Lambda^{\mathbf{m}}}{\partial\left(d \zeta_{B}\right)}\right)+\mathcal{L}_{v} \zeta_{B} \wedge \frac{\partial \Lambda^{\mathbf{m}}}{\partial\left(d \zeta_{B}\right)}
\end{aligned}
$$

Hence (45) follows from (43). Likewise from (27), (28) and $d^{2}=0$

$$
\begin{aligned}
(-1)^{p_{B}} i_{v} \zeta_{B} \wedge & d\left(\frac{\partial \Lambda^{\mathbf{m}}}{\partial \zeta_{B}}\right)+i_{v} d \zeta_{B} \wedge \frac{\partial \Lambda^{\mathbf{m}}}{\partial \zeta_{B}}+(-1)^{p_{B}+1} i_{v} d \zeta_{B} \wedge d\left(\frac{\partial \Lambda^{\mathbf{m}}}{\partial\left(d \zeta_{B}\right)}\right) \\
& =(-1)^{p_{B}} i_{v} \zeta_{B} \wedge d\left(\frac{\delta \Lambda^{\mathbf{m}}}{\delta \zeta_{B}}\right)+i_{v} d \zeta_{B} \wedge\left(\frac{\partial \Lambda^{\mathbf{m}}}{\partial \zeta_{B}}+d\left(\frac{\partial \Lambda^{\mathbf{m}}}{\partial\left(d \zeta_{B}\right)}\right)\right) \\
& =(-1)^{p_{B}} i_{v} \zeta_{B} \wedge d\left(\frac{\delta \Lambda^{\mathbf{m}}}{\delta \zeta_{B}}\right)+i_{v} d \zeta_{B} \wedge \frac{\delta \Lambda^{\mathbf{m}}}{\delta \zeta_{B}} \\
& =0
\end{aligned}
$$

hence (46) follows from (44)

\section{Lemma 13.}

$$
\mathcal{L}_{(f v)} \alpha=f \mathcal{L}_{v} \alpha+d f \wedge i_{v} \alpha \quad \text { for } \quad f \in \Gamma \Lambda^{0} M, v \in \Gamma T M \text { and } \alpha \in \Gamma \Lambda^{p} M
$$

Proof. From Cartan's identity

$$
\begin{aligned}
\mathcal{L}_{(f v)} \alpha & =i_{(f v)} d \alpha+d i_{(f v)} d \alpha=f i_{v} d \alpha+d\left(f i_{v} \alpha\right) \\
& =f i_{v} d \alpha+d f \wedge i_{v} \alpha+f d i_{v} \alpha=f \mathcal{L}_{v} \alpha+d f \wedge i_{v} \alpha
\end{aligned}
$$

Lemma 14. (159) and (60) are equivalent if $\Omega \in \Gamma \Lambda^{n} M$ is non-vanishing.

Furthermore if (60) is true for one non-vanishing $\Omega$ it is true for all non-vanishing $\Omega \in \Gamma \Lambda^{n} M$.

Proof.

$$
T(u, v) \Omega=g(\mathcal{T}(u), v) \Omega=i_{\mathcal{T}(u)} \widetilde{v} \Omega=\widetilde{v} \wedge i_{\mathcal{T}(u)} \Omega
$$

hence (59) and (60) are equivalent if $\Omega \in \Gamma \Lambda^{n} M$ is non-vanishing.

Substituting $\Omega \rightarrow \hat{\Omega}=J \Omega$ where $J \in \Gamma \Lambda^{0} M$ is non-vanishing then

$$
\widetilde{v} \wedge i_{\mathcal{T}(u)} \hat{\Omega}-\widetilde{u} \wedge i_{\mathcal{T}(v)} \hat{\Omega}=J\left(\widetilde{v} \wedge i_{\mathcal{T}(u)} \Omega-\widetilde{u} \wedge i_{\mathcal{T}(v)} \Omega\right)=0
$$


Lemma 15. Given a map $\tau: \Gamma T M \rightarrow \Gamma \Lambda^{(n-1)} M, \tau: v \mapsto \tau_{v}$, let $\mathcal{T}: \Gamma T M \rightarrow \Gamma T M$ be defined with respect to the non vanishing top form $\Omega$ via $\tau_{v}=i_{\mathcal{T}} \Omega$.

Using a coordinate frame $\left(x^{1}, \ldots, x^{n}\right)$ with $\mathcal{T}(v)^{a}=d x^{a}(\mathcal{T}(v))$ and $\Omega=d x^{1} \wedge \cdots \wedge d x^{n}$ implies

$$
\begin{gathered}
d x^{a} \wedge \tau_{v}=\mathcal{T}(v)^{a} \Omega \\
\mathcal{T}(v)^{a}=i_{\partial_{n}} \cdots i_{\partial_{1}}\left(d x^{a} \wedge \tau_{v}\right)
\end{gathered}
$$

and

$$
d \tau_{v}=\partial_{a}\left(\mathcal{T}(v)^{a}\right) \Omega
$$

Using an orthonormal coframe $\left\{e^{1}, \ldots, e^{n}\right\}$ with $\mathcal{T}(v)^{a}=e^{a}(\mathcal{T}(v))$ and $\Omega=\star 1$ then

$$
e^{a} \wedge \tau_{v}=\mathcal{T}(v)^{a} \Omega
$$

and

$$
\mathcal{T}(v)^{a}=\star^{-1}\left(d x^{a} \wedge \tau_{v}\right)
$$

Proof. Using the coordinate frame $\tau_{v}=i_{\mathcal{T}(v)} \Omega=\mathcal{T}(v)^{a} i_{\partial_{a}} \Omega$

$$
d x^{a} \wedge \tau_{v}=\mathcal{T}(v)^{b} d x^{a} \wedge i_{\partial_{b}} \Omega=\mathcal{T}(v)^{b} \delta_{b}^{a} \Omega=\mathcal{T}(v)^{a} \Omega
$$

since clearly

$$
\begin{aligned}
d x^{a} \wedge i_{\partial_{b}} \Omega & =d x^{a} \wedge i_{\partial_{b}} d x^{1} \wedge \cdots \wedge d x^{n} \\
& =(-1)^{b-1} d x^{a} \wedge d x^{1} \wedge \cdots \wedge d x^{b-1} \wedge d x^{b+1} \wedge \cdots \wedge d x^{n} \\
& =d x^{1} \wedge \cdots \wedge d x^{b-1} \wedge d x^{a} \wedge d x^{b+1} \wedge \cdots \wedge d x^{n}=\delta_{b}^{a} d x^{1} \wedge \cdots \wedge d x^{n}
\end{aligned}
$$

Hence (119) and since $i_{\partial_{n}} \cdots i_{\partial_{1}} d x^{1} \wedge \cdots \wedge d x^{n}=1$ (120). Also

$$
\begin{aligned}
d \tau_{v} & =d\left(i_{\mathcal{T}(v)} \Omega\right)=d\left(\mathcal{T}(v)^{a} i_{\partial_{a}} \Omega\right)=d\left(\mathcal{T}(v)^{a}\right) \wedge i_{\partial_{a}} \Omega+\mathcal{T}(v)^{a} d\left(i_{\partial_{a}} \Omega\right) \\
& =\partial_{b}\left(\mathcal{T}(v)^{a}\right) d x^{b} \wedge i_{\partial_{a}} \Omega=\partial_{a}\left(\mathcal{T}(v)^{a}\right) \Omega
\end{aligned}
$$

Equation (122) is proved similar to (119) and (123) is trivial.

Lemma 16. (88) holds

Proof. Using a $g$-orthonormal frame $X_{a}$ and dual coframe $e^{a}$, set $\eta_{a b}=g\left(X_{a}, X_{b}\right) \in \mathbb{R}$ and $T^{\mathcal{H}}=T_{a b} e^{a} \otimes e^{b}$. Then from (83)

$$
T_{a b} \star 1=\star T^{\mathcal{H}}\left(X_{a}, X_{b}\right)=2 \frac{\Delta \Lambda^{\mathbf{m}}}{\Delta g}:\left(e_{a} \otimes e_{b}\right)
$$

hence

$$
2 \frac{\Delta \Lambda^{\mathbf{m}}}{\Delta g}=T^{a b}(\star 1) \otimes X_{a} \otimes X_{b}
$$


From the algebraic symmetry of $T^{\mathcal{H}}, T^{a b}=T^{b a}$.

From (84) with $v=v^{a} X_{a}$

$$
\tau_{v}^{\mathcal{H}}=\star\left(T^{\mathcal{H}}(v,-)\right)=\star\left(\left(T_{a b} e^{a} \otimes e^{b}\right)(v,-)\right)=v^{a} T_{a b} \star e^{b}
$$

thus using (39) and (37) with $\Omega=\star 1$ yields

$$
\begin{aligned}
2 \mathcal{A}_{v}( & \left.\frac{\Delta \Lambda^{\mathrm{m}}}{\Delta g}, g\right)=\mathcal{A}_{v}\left(T^{a b}(\star 1) \otimes X_{a} \otimes X_{b}, e_{c} \otimes e^{c}\right) \\
& =\mathcal{A}_{v}\left(\left(X_{b}: e^{c}\right)(\star 1) \otimes\left(T^{a b} X_{a}\right), e_{c}\right)+\mathcal{A}_{v}\left(\left(T^{a b} X_{a}: e_{c}\right)(\star 1) \otimes X_{b}, e^{c}\right) \\
& =\mathcal{A}_{v}\left((\star 1) \otimes\left(T^{a c} X_{a}\right), e_{c}\right)+\mathcal{A}_{v}\left(\eta_{a c} T^{a b}(\star 1) \otimes X_{b}, e^{c}\right) \\
& =e_{c}(v) i_{T^{a c}} X_{a} \star 1+e^{c}(v) T^{a}{ }_{c} i_{X_{a}} \star 1=v_{c} T^{a c} \star e_{a}+v^{c} T^{a}{ }_{c} \star e_{a} \\
& =2 v^{c} T_{a c} \star e^{a}=2 \tau_{v}
\end{aligned}
$$

Lemma 17. (91) holds

Proof. Again using an orthonormal frame and setting $T^{\mathcal{H}}=T_{a b} e^{a} \otimes e^{b}$ and $v=v^{a} X_{a}$ as in lemma 16 and using (39), (37), the algebraic symmetry of $T^{\mathcal{H}}$ and (90) yields

$$
\begin{aligned}
2 \mathcal{B}_{v}( & \left.\frac{\Delta \Lambda^{\mathbf{m}}}{\Delta g}, g\right)=\mathcal{B}_{v}\left(T^{a b}(\star 1) \otimes X_{a} \otimes X_{b}, e_{c} \otimes e^{c}\right) \\
= & \mathcal{B}_{v}\left(\left(X_{b}: e^{c}\right)(\star 1) \otimes\left(T^{a b} X_{a}\right), e_{c}\right)+\mathcal{B}_{v}\left(\left(T^{a b} X_{a}: e_{c}\right)(\star 1) \otimes X_{b}, e^{c}\right) \\
= & \mathcal{B}_{v}\left((\star 1) \otimes\left(T^{a c} X_{a}\right), e_{c}\right)+\mathcal{B}_{v}\left(T_{c}^{b}(\star 1) \otimes X_{b}, e^{c}\right) \\
= & (-1)^{n-1} T^{a c}\left(\star e_{a}\right) \wedge i_{v} d e_{c}-d\left(T^{a c} \star e_{a}\right) \wedge i_{v} e_{c} \\
& \quad+(-1)^{n-1} T_{c}^{b}\left(\star e_{b}\right) \wedge i_{v} d e^{c}-d\left(T_{c}^{b} \star e_{b}\right) \wedge i_{v} e^{c} \\
= & 2\left(i_{v} d e^{c} \wedge T_{a c}\left(\star e^{a}\right)-d\left(T_{a c} \star e^{a}\right) \wedge i_{v} e^{c}\right) \\
= & -2\left(v^{c} d\left(\tau_{X_{c}}^{\mathcal{H}}\right)-i_{v} d e^{c} \wedge \tau_{X_{c}}^{\mathcal{H}}\right)=-2\left(D \tau^{\mathcal{H}}\right)_{v}
\end{aligned}
$$

The remainder of this appendix is to derive (52) in the text. Since it is convenient not to use the summation convention for multiindex summations, the following lemmas require the introduction of some new notation.

Given positive integers $n$ and $r$, where $r \leq n$ then denote $\operatorname{Inc}(r, n)$ as the set of lists of $r$ elements, which are increasing

$$
\operatorname{Inc}(r, n)=\left\{I:\{1, \ldots, r\} \rightarrow\{1, \ldots, n\} \mid I_{1}<I_{2}<\cdots<I_{r}\right\}
$$

We use the notation $I \in \operatorname{Inc}(r, n)$ with $\operatorname{len}(I)=r$. For example $\operatorname{Inc}(2,3)=\{[1,2],[1,3],[2,3]\}$. The sum over the set $\operatorname{Inc}(r, n)$ is then written

$$
\sum_{I \in \operatorname{Inc}(r, n)}=\sum_{1 \leq I_{1}<I_{2}<\cdots<I_{r} \leq n}=\sum_{I_{1}=1}^{n} \sum_{I_{2}=I_{1}+1}^{n} \cdots \sum_{I_{r}=I_{r-1}+1}^{n}
$$


Given positive integers $n$ and $r$, Free $(r, n)$ is the set of lists of $r$ elements not necessarily increasing

$$
\operatorname{Free}(r, n)=\{\hat{I}:\{1, \ldots, r\} \rightarrow\{1, \ldots, n\}\}
$$

We use the notation $\hat{I} \in \operatorname{Free}(r, n)$. For example

$$
\operatorname{Free}(2,3)=\{[1,1],[1,2],[1,3],[2,1],[2,2],[2,3],[3,1],[3,2],[3,3]\}
$$

The sum over the set Free $(r, n)$ is then written

$$
\sum_{I \in \operatorname{Free}(r, n)}=\sum_{1 \leq I_{1}, \ldots, I_{r} \leq n}=\sum_{I_{1}=1}^{n} \sum_{I_{2}=1}^{n} \cdots \sum_{I_{r}=1}^{n}
$$

Let $\left\{e^{a}, a=1, \ldots, n\right\}$ be a coframe (not necessarily orthonormal) and $X_{a}$ its dual. Let

$$
e^{I}=e^{I_{1}} \wedge \cdots \wedge e^{I_{r}}
$$

and let $i_{a}=i_{X_{a}}$ and

$$
i_{I}=i_{I_{r}} \cdots i_{I_{1}}
$$

Clearly for $\alpha_{I}$ antisymmetric then

$$
\sum_{I \in \operatorname{Inc}(r, n)} \alpha_{I} e^{I}=\frac{1}{r !} \sum_{\hat{I} \in \operatorname{Free}(r, n)} \alpha_{I} e^{I}
$$

For free list $\hat{I} \in \operatorname{Free}(r, n)$ let $\epsilon(\hat{I})$ is the signature of $\hat{I}$ and $\operatorname{Inc}(\hat{I})$ is the increasing form of $\hat{I}$. Here $\epsilon(\hat{I})=0$ if $\hat{I}$ contains repeated indices. Thus

$$
e^{\hat{I}}=\epsilon(\hat{I}) e^{\operatorname{Inc}(\hat{I})}
$$

For free lists $\hat{I}, \hat{J} \in \operatorname{Free}(r, n)$ let

$$
\delta_{\hat{J}}^{\hat{I}}= \begin{cases}\epsilon(\hat{I}) \epsilon(\hat{J}) & \text { if } \operatorname{Inc}(\hat{I})=\operatorname{Inc}(\hat{J}) \\ 0 & \text { Otherwise }\end{cases}
$$

Thus for increasing lists $I, J \in \operatorname{Inc}(r, n)$ then $\delta_{J}^{I}=1$ if $I=J$ and $\delta_{J}^{I}=0$ if $I \neq J$.

We use concatenation to represent the combining of lists, so that if $\hat{I} \in \operatorname{Free}(r, n)$ and $\hat{J} \in \operatorname{Free}(s, n)$ then $\hat{I} \hat{J} \in \operatorname{Free}(r+s, n)$ in the natural way, i.e.

$$
(\hat{I} \hat{J})_{\mu}= \begin{cases}\hat{I}_{\mu} & \text { if } \quad \mu \leq r \\ \hat{J}_{\mu-r} & \text { if } \quad \mu>r\end{cases}
$$

Likewise if $a \in\{1, \ldots, n\}$ and $I \in \operatorname{Inc}(r, n)$ then $a I \in \operatorname{Free}(r+1, n)$. We use the backslash to represent the removal of an element from a list. That is for $\hat{J} \in \operatorname{Free}(r, n)$ and $1 \leq s \leq r$ then $J \backslash J_{s} \in \operatorname{Free}(r-1, n)$ is given by

$$
\left(\hat{J} \backslash J_{s}\right)_{\mu}= \begin{cases}\hat{J}_{\mu} & \text { if } \quad \mu<s \\ \hat{J}_{\mu+1} & \text { if } \mu \geq s\end{cases}
$$




\section{Lemma 18.}

$$
e^{a} \wedge i_{a} \alpha=p \alpha \quad \text { for } \quad \alpha=\Gamma \Lambda^{p} M
$$

Proof. Clearly true for $p=0$. By induction, assume true for $\alpha \in \Gamma \Lambda^{p} M$ let $\beta \in \Gamma \Lambda^{1} M$.

$$
\begin{aligned}
e^{a} \wedge i_{a}(\beta \wedge \alpha) & =e^{a} \wedge\left(i_{a} \beta \wedge \alpha\right)-e^{a} \wedge\left(\beta \wedge i_{a} \alpha\right)=\beta \wedge \alpha+\beta \wedge e^{a} \wedge i_{a} \alpha \\
& =(p+1) \beta \wedge \alpha
\end{aligned}
$$

Lemma 19.

$$
\sum_{I \in \operatorname{Inc}(r, n)} i_{I}\left(e^{I} \wedge \alpha\right)=\frac{1}{r !} \sum_{\hat{I} \in \operatorname{Free}(r, n)} i_{\hat{I}}\left(e^{\hat{I}} \wedge \alpha\right)=\left(\begin{array}{c}
n-p \\
r
\end{array}\right) \alpha \quad \text { for } \quad \alpha=\Gamma \Lambda^{p} M
$$

Proof. Clearly the first two expression are equivalent.

Consider $r=1$ then

$$
i_{a}\left(e^{a} \wedge \alpha\right)=\delta_{a}^{a} \alpha-e^{a} \wedge i_{a} \alpha=(n-p) \alpha
$$

Then the left hand side of (128) becomes

$$
\begin{aligned}
\frac{1}{r !} \sum_{\hat{I} \in \operatorname{Free}(r, n)} i_{\hat{I}}\left(e^{\hat{I}} \wedge \alpha\right) & =\frac{1}{r !} i_{\hat{I}_{r}} \cdots i_{\hat{I}_{1}}\left(e^{\hat{I}_{1}} \wedge \cdots \wedge e^{\hat{I}_{r}} \wedge \alpha\right) \\
& =\frac{1}{r !}(n-(p+r-1)) i_{\hat{I}_{r}} \cdots i_{\hat{I}_{2}}\left(e^{\hat{I}_{2}} \wedge \cdots \wedge e^{\hat{I}_{r}} \wedge \alpha\right) \\
& =\cdots=\frac{1}{r !}(n-(p+r-1)) \cdots(n-p) \alpha \\
& =\frac{1}{r !}(n-p-r+1) \cdots(n-p) \alpha=\frac{(n-p) !}{r !(n-p-r) !} \alpha
\end{aligned}
$$

Corollary 20. If $\operatorname{deg}\left(e^{I} \wedge \alpha\right)=n$ then

$$
\sum_{I \in \operatorname{Inc}(r, n)} i_{I}\left(e^{I} \wedge \alpha\right)=\alpha
$$

Proof. Since $n-p=r$.

Lemma 21. For increasing lists $I, J \in \operatorname{Inc}(r, n)$ and $\Omega \in \Gamma \Lambda^{n} M$ then

$$
e^{I} \wedge i_{J} \Omega=\Omega \delta_{J}^{I}
$$

Proof. In the proof of lemma [15] we show

$$
e^{a} \wedge i_{b} \Omega=\delta_{b}^{a} \Omega
$$

So

$$
\begin{aligned}
e^{I} \wedge i_{J} \Omega & =e^{I_{1}} \wedge \cdots \wedge e^{I_{r}} \wedge i_{J_{r}} \cdots i_{J_{1}} \Omega=e^{I_{1}} \wedge \cdots \wedge e^{I_{r-1}} \wedge i_{J_{r-1}} \cdots i_{J_{1}} \Omega \delta_{J_{r}}^{I_{r}} \\
& =\Omega \delta_{J_{r}}^{I_{r}} \delta_{J_{r-1}}^{I_{r-1}} \cdots \delta_{J_{1}}^{I_{1}}=\Omega \delta_{J}^{I}
\end{aligned}
$$


Corollary 22. For free lists $\hat{I}, \hat{J} \in \operatorname{Free}(r, n)$ and $\Omega \in \Gamma \Lambda^{n} M$ then

$$
e^{\hat{I}} \wedge i_{\hat{J}} \Omega=\Omega \delta_{\hat{J}}^{\hat{I}}
$$

Proof.

$$
e^{\hat{I}} \wedge i_{\hat{J}} \Omega=\epsilon(\hat{I}) \epsilon(\hat{J}) e^{\operatorname{Inc}(\hat{I})} \wedge i_{\operatorname{Inc}(\hat{J})} \Omega=\epsilon(\hat{I}) \epsilon(\hat{J}) \delta_{\operatorname{Inc}(\hat{J})}^{\operatorname{Inc}(\hat{I})} \Omega=\delta_{\hat{J}}^{\hat{I}} \Omega
$$

Lemma 23. Let $I \in \operatorname{Inc}(r, n)$ and $J \in \operatorname{Inc}(r+1, n)$ and $\Omega \in \Gamma \Lambda^{n} M$ then

$$
e^{I} \wedge i_{J} \Omega=\sum_{s=1}^{r+1}(-1)^{s-1} \delta_{J \backslash J_{s}}^{I} i_{J_{s}} \Omega
$$

Proof. First observe that for the left hand side of (132) to be non zero then $I \subset J$. Since $I$ and $J$ are increasing then $J_{r+1} \neq I_{s}$ for $s=1, \ldots, r-1$. Thus there are two cases, either $J_{r+1}=I_{r}$ or $J_{r+1}$ does not equal any $I_{s}$. I.e.

$$
i_{J_{r+1}} e^{I}=(-1)^{r-1} \delta_{J_{r+1}}^{I_{r}} e^{I \backslash I_{r}}
$$

By induction on $r$. Clearly true when $I \in \operatorname{Inc}(0, n)$. Assume true when $I \in \operatorname{Inc}(r-1, n)$ then

$$
\begin{aligned}
e^{I} \wedge i_{J} \Omega= & e^{I} \wedge i_{J_{r+1}} i_{J \backslash J_{r+1}} \Omega \\
= & (-1)^{r} i_{J_{r+1}}\left(e^{I} \wedge i_{J \backslash J_{r+1}} \Omega\right)+(-1)^{r-1} i_{J_{r+1}} e^{I} \wedge i_{J \backslash J_{r+1}} \Omega \\
& \text { from (130) and (133) } \\
= & (-1)^{r} \delta_{J \backslash J_{r+1}}^{I} i_{J_{r+1}} \Omega+\delta_{J_{r+1}}^{I_{r}} e^{I \backslash I_{r}} \wedge i_{J \backslash J_{r+1}} \Omega \\
\text { from induction hypot } & (-1)^{r} \delta_{J \backslash J_{r+1}}^{I} i_{J_{r+1}} \Omega+\delta_{J_{r+1}}^{I_{r}}\left(\sum_{s=1}^{r}(-1)^{s-1} \delta_{J \backslash J_{r+1} \backslash J_{s}}^{I \backslash I_{J_{s}}} \Omega\right) \\
= & (-1)^{r} \delta_{J \backslash J_{r+1}}^{I} i_{J_{r+1}} \Omega+\sum_{s=1}^{r}(-1)^{s-1} \delta_{J \backslash J_{s}}^{I} i_{J_{s}} \Omega \\
= & \sum_{s=1}^{r+1}(-1)^{s-1} \delta_{J \backslash J_{s}}^{I} i_{J_{s}} \Omega
\end{aligned}
$$$$
\text { from induction hypothesis }
$$

Lemma 24. Let $\mathcal{V}^{J}$ be a multiindex object where $J \in \operatorname{Inc}(r+1, n)$ and let $I \in \operatorname{Inc}(r, n)$ and $\Omega \in \Gamma \Lambda^{n} M$ then

$$
\sum_{J \in \operatorname{Inc}(r+1, n)} \mathcal{V}^{J} \sum_{s=1}^{r+1}(-1)^{s-1} \delta_{J \backslash J_{s}}^{I} i_{J_{s}} \Omega=\sum_{a=1}^{n} \mathcal{V}^{\operatorname{Inc}(a I)} \epsilon(a I) i_{a} \Omega
$$


Proof. The only values of $J$ such that $\delta_{J \backslash J_{s}}^{I}=1$ are when $J=\operatorname{Inc}(a I)$ for some $a$. In this case one value of $s$ is $\delta_{J \backslash J_{s}}^{I}=1$ and

$$
(-1)^{s-1}=\epsilon(a I)
$$

Hence result

Corollary 25. Let $\mathcal{V}^{\hat{J}}$ be an antisymmetric multiindex object where $\hat{J} \in \operatorname{Free}(r+1, n)$ and let $I \in \operatorname{Inc}(r, n)$ and $\Omega \in \Gamma \Lambda^{n} M$ then

$$
\sum_{J \in \operatorname{Inc}(r+1, n)} \mathcal{V}^{J} \sum_{s=1}^{r+1}(-1)^{s-1} \delta_{J \backslash J_{s}}^{I} i_{J_{s}} \Omega=\sum_{a=1}^{n} \mathcal{V}^{a I} i_{a} \Omega
$$

Proof. Follows since

$$
\mathcal{V}^{\operatorname{Inc}(a I)} \epsilon(a I)=\mathcal{V}^{a I}
$$

Corollary 26. Let $\mathcal{V}^{\hat{J}}$ and $\alpha_{\hat{I}}$ be antisymmetric multiindex objects where $\hat{J} \in \operatorname{Free}(r+1, n)$ and $\hat{I} \in \operatorname{Free}(r, n)$ and let $\Omega \in \Gamma \Lambda^{n} M$ then

$$
\sum_{I \in \operatorname{Inc}(r, n)} \sum_{J \in \operatorname{Inc}(r+1, n)} \mathcal{V}^{J} \alpha_{I} e^{I} \wedge i_{J} \Omega=\sum_{I \in \operatorname{Inc}(r, n)} \sum_{a=1}^{n} \alpha_{I} \mathcal{V}^{a I} i_{a} \Omega
$$

Lemma 27. (52) holds.

Proof. From (49)

$$
\mathcal{N}_{\partial_{a}}=i_{\mathcal{T} \mathcal{N}\left(\partial_{a}\right)} \Omega=\mathcal{T}^{\mathcal{N}}{ }_{a}^{b} i_{b} \Omega
$$

Let $\operatorname{deg}\left(d \zeta_{B}\right)=r+1$ and $d \zeta_{B}=\sum_{I \in \operatorname{Inc}(r+1, n)}\left(d \zeta_{B}\right)_{I} d x^{I}$ and $J \in \operatorname{Inc}(r+1, n)$ then from (25)

$$
\begin{aligned}
d x^{J} \wedge \frac{\partial \Lambda^{\mathbf{m}}}{\partial\left(d \zeta_{B}\right)} & =\left.\frac{d}{d \varepsilon}\right|_{\varepsilon=0} \Lambda^{\mathbf{m}}\left(\ldots, d \zeta_{B}+\epsilon d x^{J}, \ldots\right) \\
& =\left.\frac{d}{d \varepsilon}\right|_{\varepsilon=0} \mathcal{S}\left(\ldots,\left(d \zeta_{B}\right)_{J}+\epsilon, \ldots\right) \Omega=\frac{\partial \mathcal{S}}{\partial\left(d \zeta_{B}\right)_{J}} \Omega
\end{aligned}
$$

Hence from (129)

$$
\sum_{J \in \operatorname{Inc}(r+1, n)} \frac{\partial \mathcal{S}}{\partial\left(d \zeta_{B}\right)_{J}} i_{J} \Omega=\sum_{J \in \operatorname{Inc}(r+1, n)} i_{J}\left(d x^{J} \wedge \frac{\partial \Lambda^{\mathbf{m}}}{\partial\left(d \zeta_{B}\right)}\right)=\frac{\partial \Lambda^{\mathbf{m}}}{\partial\left(d \zeta_{B}\right)}
$$


From (47)

$$
\begin{aligned}
\mathcal{N}_{\partial_{a}} & =i_{a} \Lambda^{\mathbf{m}}-\sum_{B=1}^{Q} \mathcal{L}_{\partial_{a}} \zeta_{B} \wedge \frac{\partial \Lambda^{\mathbf{m}}}{\partial\left(d \zeta_{B}\right)} \\
& =i_{a}(\mathcal{S} \Omega)-\sum_{B=1}^{Q} \sum_{I \in \operatorname{Inc}(r, n)} \sum_{J \in \operatorname{Inc}(r+1, n)} \mathcal{L}_{\partial_{a}}\left(\zeta_{B I} d x^{I}\right) \wedge \frac{\partial \mathcal{S}}{\partial\left(d \zeta_{B}\right)_{J}} i_{J} \Omega \\
& =\mathcal{S} i_{a} \Omega-\sum_{B=1}^{Q} \sum_{I \in \operatorname{Inc}(r, n)} \sum_{J \in \operatorname{Inc}(r+1, n)} \partial_{a}\left(\zeta_{B I}\right) \frac{\partial \mathcal{S}}{\partial\left(d \zeta_{B}\right)_{J}} d x^{I} \wedge i_{J} \Omega \\
& =\delta_{a}^{b} \mathcal{S} i_{b} \Omega-\sum_{B=1}^{Q} \sum_{I \in \operatorname{Inc}(r, n)} \partial_{a}\left(\zeta_{B I}\right) \frac{\partial \mathcal{S}}{\partial\left(d \zeta_{B}\right)_{b I}} i_{b} \Omega
\end{aligned}
$$

Hence

$$
\left(\mathcal{T}^{\mathcal{N}}{ }_{a}^{b}-\delta_{a}^{b} \mathcal{S}+\sum_{B=1}^{Q} \sum_{I \in \operatorname{Inc}(r, n)} \partial_{a}\left(\zeta_{B I}\right) \frac{\partial \mathcal{S}}{\partial\left(d \zeta_{B}\right)_{b I}}\right) i_{b} \Omega=0
$$

Thus

$$
\begin{aligned}
0 & =\left(\mathcal{T}^{\mathcal{N}}{ }_{a}^{b}-\delta_{a}^{b} \mathcal{S}+\sum_{B=1}^{Q} \sum_{I \in \operatorname{Inc}(r, n)} \partial_{a}\left(\zeta_{B I}\right) \frac{\partial \mathcal{S}}{\partial\left(d \zeta_{B}\right)_{b I}}\right) e^{c} \wedge i_{b} \Omega \\
& =\left(\mathcal{T}^{\mathcal{N}}{ }_{a}{ }^{b}-\delta_{a}^{b} \mathcal{S}+\sum_{B=1}^{Q} \sum_{I \in \operatorname{Inc}(r, n)} \partial_{a}\left(\zeta_{B I}\right) \frac{\partial \mathcal{S}}{\partial\left(d \zeta_{B}\right)_{b I}}\right) \delta_{b}^{c} \Omega
\end{aligned}
$$

giving

$$
\mathcal{T}^{\mathcal{N}}{ }_{a}{ }^{b}=\delta_{a}^{b} \mathcal{S}-\sum_{B=1}^{Q} \sum_{I \in \operatorname{Inc}(r, n)} \partial_{a}\left(\zeta_{B I}\right) \frac{\partial \mathcal{S}}{\partial\left(d \zeta_{B}\right)_{b I}}
$$

Now since

$$
d \zeta_{B}=\sum_{I \in \operatorname{Inc}(r, n)} d\left(\zeta_{B I} d x^{I}\right)=\sum_{I \in \operatorname{Inc}(r, n)} \partial_{b} \zeta_{B I} d x^{b I}=\sum_{I \in \operatorname{Inc}(r, n)} \epsilon(b I) \partial_{b} \zeta_{B I} d x^{\operatorname{Inc}(b I)}
$$

then

$$
\frac{\partial \mathcal{S}}{\partial\left(d \zeta_{B}\right)_{b I}}=\epsilon(b I) \frac{\partial \mathcal{S}}{\partial\left(d \zeta_{B}\right)_{\operatorname{Inc}(b I)}}=\epsilon(b I)^{2} \frac{\partial \mathcal{S}}{\partial\left(\partial_{b} \zeta_{B I}\right)}=\frac{\partial \mathcal{S}}{\partial\left(\partial_{b} \zeta_{B I}\right)}
$$

and

$$
\mathcal{T}^{\mathcal{N}}{ }_{a}^{b}=\delta_{a}^{b} \mathcal{S}-\sum_{B=1}^{Q} \sum_{I \in \operatorname{Inc}(r, n)} \partial_{a}\left(\zeta_{B I}\right) \frac{\partial \mathcal{S}}{\partial\left(\partial_{b} \zeta_{B I}\right)}
$$

I.e. (52). 


\section{B Computational method for calculating Gateaux deriva- tives}

In any local frame $\left\{X_{a}\right\}$ with dual co-frame $\left\{e^{b}\right\}$ let $\Lambda^{\mathbf{m}}(Z, \ldots) \in \Gamma \Lambda^{n} M$ depend on the tensor $Z=Z^{a}{ }_{b} X_{a} \otimes e^{b}$. Relative to any chosen top-form $\Omega$ write $\Lambda^{\mathrm{m}}(Z, \ldots)=\mathcal{S}\left(Z_{b}^{a}, \ldots\right) \Omega$ where $\mathcal{S} \in \Gamma \Lambda^{0} M$ and $\Omega$ is independent of $Z$. Thus $\mathcal{S}\left(Z^{a}{ }_{b}, \ldots\right)$ depends on the $n^{2}$ variables $Z_{b}^{a}$ with $n^{2}$ derivatives $\frac{\partial \mathcal{S}}{\partial Z^{a}{ }_{b}}$. In this case

$$
\begin{aligned}
\frac{\Delta \Lambda^{\mathbf{m}}}{\Delta Z}: Y & =\left.\frac{d}{d \varepsilon}\right|_{\varepsilon=0} \Lambda^{\mathbf{m}}(Z+\varepsilon Y, \ldots)=\left.\frac{d}{d \varepsilon}\right|_{\varepsilon=0} \mathcal{S}\left(Z_{b}^{a}+\varepsilon Y_{b}^{a}, \ldots\right) \Omega=\frac{\partial \mathcal{S}}{\partial Z_{b}^{a}} Y_{b}^{a} \Omega \\
& =\frac{\partial \mathcal{S}}{\partial Z^{a}{ }_{b}} \Omega \otimes e^{a} \otimes X_{b}: Y
\end{aligned}
$$

hence

$$
\frac{\Delta \Lambda^{\mathrm{m}}}{\Delta Z}=\frac{\partial \mathcal{S}}{\partial Z^{a} b} \Omega \otimes e^{a} \otimes X_{b}
$$

For example if $\mathcal{S}$ is such that $\Lambda^{\mathbf{m}}(Z, \alpha, \beta, u, v)=Z(\alpha, v) Z(\beta, u) \Omega$ for any 1-form fields $\alpha, \beta \in \Gamma \Lambda^{1} M$ and vector fields $u, v \in \Gamma T M$ then

$$
\mathcal{S}\left(Z^{a}{ }_{b} \alpha, \beta, u, v\right)=Z^{a}{ }_{b} Z^{c}{ }_{d} \alpha_{a} v^{b} \beta_{c} u^{d}
$$

and it follows from the definition that

$$
\begin{aligned}
\frac{\Delta \Lambda^{\mathrm{m}}}{\Delta Z} & =\alpha_{a} v^{d} \beta_{c} u^{b} Z^{c}{ }_{d} \Omega \otimes e^{a} \otimes X_{b}+\alpha_{c} v^{d} \beta_{a} u^{b} Z^{c}{ }_{d} \Omega \otimes e^{a} \otimes X_{b} \\
& =Z(\beta, u) \Omega \otimes \alpha \otimes v+Z(\alpha, v) \Omega \otimes \beta \otimes u
\end{aligned}
$$

